\title{
THE LEPIDOPTERA OF BUCHAREST AND ITS SURROUNDINGS (ROMANIA)
}

\author{
LEVENTE SZÉKELY
}

\begin{abstract}
This study presents a synthesis of the current knowledge regarding the Lepidoptera fauna of Bucharest and the surrounding areas within a distance up to 50 kilometers around the Romanian capital. Data about the fauna composition are presented: the results of the research work beginning with the end of the $19^{\text {th }}$ century, as well the results of the research work carried out in the last 15 years. The research initiated and done by the author himself, led to the identification of 180 species which were unknown in the past. Even if the natural habitats from this region have undergone through radical changes in the $20^{\text {th }}$ century, the area still preserves a quite rich and interesting Lepidoptera fauna. The forests provide shelter to rich populations of the hawk moth Dolbina elegans A. Bang-Haas, 1912, one of the rarest Sphingidae in Europe, and some other species with high faunistical and zoogeographical value as: Noctua haywardi (Tams, 1926) (it is new record for the Romanian fauna from this area), Catocala dilecta (Hübner, 1808), Tarachidia candefacta (Hübner, [1831]), Chrysodeixis chalcites (Esper, [1789]), Aedia leucomelas (Linnaeus, 1758), and Hecatera cappa (Hübner, [1809]). We also present and discuss the current status of the protected Lepidoptera species from the surroundings of the Romanian capital for the first time.
\end{abstract}

Résumé. Ce travail représente une synthèse des connaissances actuelles concernant la faune de lépidoptères de Bucarest et de ses zones limitrophes sur un rayon de $50 \mathrm{~km}$ autour de la capitale de la Roumanie. On présente toutes les données sur la composition de la faune de lépidoptères publiées à partir de la fin du XIX-ème siècle, ainsi que les résultats des dernières 15 années. Les recherches faites par l'auteur après 1966 ajoutent 180 nouvelles espèces pour la région. Bien que le cadre naturel de cette région ait été radicalement changé au cours du XX-ème siècle, elle conserve une assez riche et intéressante faune. Les forets abrîtent de nombreux exemplaires du sfingide Dolbina elegans A. Bang-Haas, 1912, l'une des plus rares espèces de sfingide de l'Europe, ainsi que d'autres espèces de valeur faunistique et zoogéographique comme Noctua haywardi (Tams, 1926) (signalée de cette zone comme nouvelle pour la faune de Roumanie), Catocala dilecta (Hübner, 1808), Tarachidia candefacta (Hübner, [1831]), Chrysodeixix chalcites (Esper, [1789]), Aedia leucomelas (Linnaeus, 1758) et Hecatera cappa (Hübner, [1809]). On présente pour la première fois la situation actuelle des espèces protégées par la législation européenne et nationale de la zone de la capitale roumaine.

Key words: Lepidoptera, fauna, old records, new records, Bucharest, Romania.

\section{INTRODUCTION}

The surroundings of the large European cities and especially the surroundings of the capitals have suffered major changes of natural scenery in the $20^{\text {th }}$ century, that affected the Lepidoptera fauna (butterflies and moths), too. Romania was not an exception since its capital and the surrounding areas have undergone radical changes in the last 100 years. The study of the Lepidoptera fauna has begun in this region around 1880 and the first published studies about the Lepidoptera fauna of 
Romania have partially referred to this area too (Caradja, 1896; Montandon, 1900; Abafi-Aigner, 1901; Salay, 1910). They represented the only reliable knowledge about the fauna of Bucharest ("București" in Romanian). In those times, however, the surroundings of the Romanian capital were very different compared to what can be seen nowadays. There were much wider forested areas, wetlands, moors and large areas of land were farmed by traditional means. Even in the inner city there was a much richer Lepidoptera fauna than nowadays, because in a city made up mainly of gardens and houses, as Bucharest used to be in those times, the butterfly and moth species assemblages were different compared to the nowadays fauna of apartmentbuilding districts.

The scenery of Bucharest and its surroundings has radically changed after the $1950 \mathrm{~s}$, as soon as the land areas were brought under intensive farming, the wetlands under drainage work, the herbicides and insecticides were used on a large scale, and transports and urbanization were developed (Székely, 1994). The research work started by A. Ostrogovich upon the Lepidoptera fauna until 1935 and the research started by the eminent entomologist A. Popescu-Gorj in 1938 (Popescu, 1938, 1939; Popescu-Gorj, 1960, 1964) has practically ended the study of Lepidoptera-fauna from Bucharest and its surroundings from that period. Due to the alterations of natural environment, the research work has entered a period of decline after 1970, and many of the species which had been recorded 50-100 years before, were considered as "probably extinct".

The aim of this work is to demonstrate the contrary. The researches from the period 1996-2010 have brought new results and, based on these new data, we can conclude that the sorroundings of Bucharest (Fig. 1) still preserve, even at present, a fairly rich and varied entomofauna. A lot of species that were considered "probably extinct" were recorded again. 180 species are completely new to this region and 55 species are new to Wallachia. Noctua haywardi (Tams, 1926) was reported as a new record for the entomofauna of Romania based on material collected in this region (Székely \& Stanciu, 2002). The forests surrounding the capital city provide shelter to rich populations of Dolbina elegans A. Bang-Haas, 1912, one of the rarest hawk moths of the European Lepidoptera fauna and 17 species would be welcome for European and Natural laws.

\section{MATERIAL AND METHODS}

The material was collected by aiming the still forested areas. The diurnal collecting was done with the insect net, while the nocturnal species were captured by using a $125 \mathrm{~W}$ mercury vapor bulb placed in front of a white sheet. In addition, three to five portable light traps with $8 \mathrm{~W}$ white and black light tubes were used during each collecting event. 


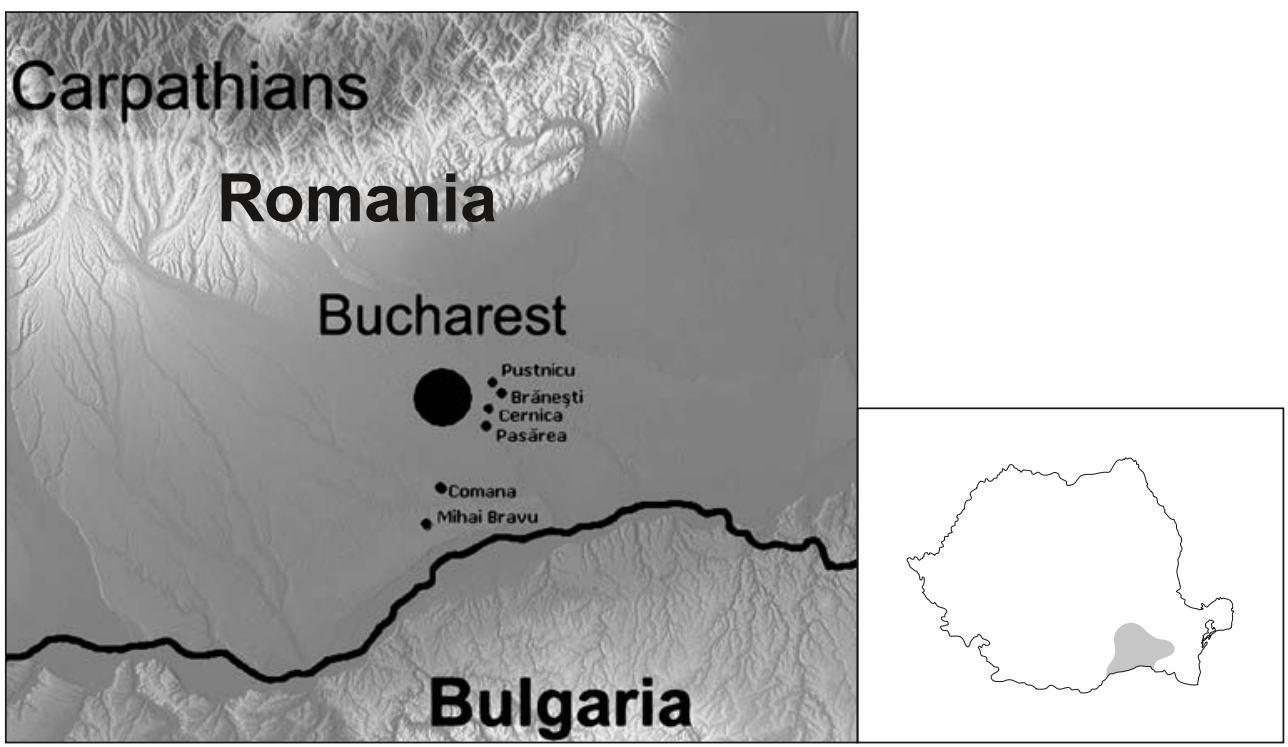

Fig. 1 - Localities investigated for this study in the period 1996-2010.

Two - three centuries ago, forests covered large territories in the surroundings of Bucharest. The famouse "Codrii Vlăsiei - Vlăsiei Forest", to which the aforementioned forested areas pertained to, were gradually clear cut. Now such forested areas form island-like patches, on restrained territories of forest vegetation. The climate is continental, with very hot summers and frosty winters. The yearly average temperature is $10-11^{\circ} \mathrm{C}$. The highest average temperatures occur in July when they exceed $23^{\circ} \mathrm{C}$. The yearly rainfall is around $500-600 \mathrm{~mm}$ with mostly heavy summer showers. The winter is characterized by cold air intrusions but snowfalls are scarce.

Wallachia ("Muntenia" in Romanian) is a historical region in southern Romania. Its boundaries are the Carpathian Mountains in north (which delimits of Transylvania), in west the Olt River (which separates it from Oltenia), in south and east the Danube River (which delimits Bulgaria and Dobrogea). Dobrogea is a historical province of Romania located east of Wallachia, and Oltenia another historical province situated west of Wallachia.

The Romanian Plain (Câmpia Română) is located in southern Romania. Part of the historical region of Wallachia, it is bordered by the Danube River in the east, south and west, and by the Getic Plateau in the north. Bucharest, the capital of Romania, is located in the central part of the Romanian Plain. It is contiguous to the south with the Danubian Plain, in Bulgaria.

This work comprises the data gathered during the systematic collection work done in different forested areas that surround the capital: Brăneşti (the Bridge of Brăneşti, the former small railway station CFR-Cozieni), Pasărea (the forest district 
and the vicinity of the railway station), Comana (Comana Forest), Cernica (the vicinity of the monastery) and Pustnicu (Pustnicu Forest). In these areas collecting activities were done during different periods of the year (May - October) between 1996 - 2010 (Fig. 1). Occasional collecting has been carried out at: Călugăreni, Jilava, Pădurea Buda (Buda Forest). The source of the systematic order is „The Lepidoptera of Europe" (Karlsholt \& Razowsky, 1996).

List of collecting localities with geographical coordinates (Fig. 1):

Pustnicu - forest in Ilfov county $\left(44^{\circ} 53^{\prime} \mathrm{N} / 26^{\circ} 09^{\prime} \mathrm{E}\right)$

Brăneşti - village in Ilfov county $\left(44^{\circ} 45^{\prime} \mathrm{N} / 26^{\circ} 33^{\prime} \mathrm{E}\right)$

Cernica - village and forest in Ilfov county $\left(44^{\circ} 40^{\prime} \mathrm{N} / 26^{\circ} 26^{\prime} \mathrm{E}\right)$

Pasărea - village and forest in Ilfov county $\left(44^{\circ} 28^{\prime} \mathrm{N} / 26^{\circ} 19^{\prime} \mathrm{E}\right)$

Comana - village and forest in Giurgiu county $\left(44^{\circ} 17^{\prime} \mathrm{N} / 26^{\circ} 13^{\prime} \mathrm{E}\right)$

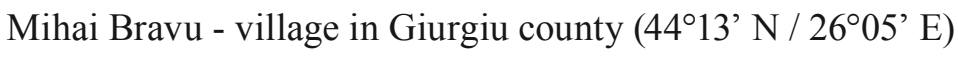

Abbreviations:

*- new species for Bucharest and its surroundings

**- older records, considered doubtful at present

very rare (1-3 specimens/collecting day or night)

rare (4-9 specimens/collecting day or night)

common (10-29 specimens/collecting day or night)

very common (more than 30 specimens/collecting day or night).

Păd. = forest ("pădure" in Romanian)

$\hat{\jmath}=$ male, + q female

\section{RESULTS}

The results of the research work done between 1996-2010 comprise for now about 520 species. Considering the whole amount of literature data, the area of Bucharest and its surroundings can be considered as being intensely entomologically researched, since 822 species of Lepidoptera (590 species of Macrolepidoptera and 232 species of Microlepidoptera) have been recorded up to the present day.

From the impressive amount of 822 species we can find data in literature referring to 632 of them. Therefore, the faunistic list of Lepidoptera of Bucharest and its surroundings has increased by 180 species which hadn't been reported in the past.

Concerning the butterflies (Papilionoidea \& Hesperioidea), over 50\% of the species that have been recorded in the past were not found again. One should however not forget the fact that 100 years ago researchers investigated mostly the butterfly-fauna. Another aspect to be taken into consideration is the fact that the habitats of these species were altered considerably during the last 100 years since these species favour open areas, forest selvages and clearings, all being habitats which were most severely altered by man. 
FAUNISTIC LIST OF LEPIDOPTERA

Family Lasiocampidae

Eriogaster lanestris (Linnaeus, 1758): Bucureşti, 28.III.1895, coll. Salay, (PopescuGorj, 1964).

Malacosoma neustria (Linnaeus, 1758): Brăneşti, Pasărea, Pustnicu, Comana, 12.VII.9.VIII.1996-2008, common. Further records: Bucureşti, Comana (Montandon, 1900; Popescu-Gorj, 1964).

Lasiocampa trifolii ([Denis \& Schiffermüller], 1775): Comana, Ţigăneşti, 1915-1916 (Popescu-Gorj, 1964).

Lasiocampa quercus (Linnaeus, 1758)*: Pasărea, Păd. Buda, 12.VII.-1.VIII.1997-99, rare.

Macrothylacia rubi (Linnaeus, 1758): Comana, Chitila, Bucureşti (Popescu-Gorj, 1964).

Euthrix potatoria (Linnaeus, 1758)**: Bucureşti, 6.VI.1892, coll. Salay (PopescuGorj, 1964).

Phyllodesma tremulifolia (Hübner, 1810): Comana, Mihai Bravu, 12-13.VII.1996-97, rare. Further records: Bucureşti, Comana (Popescu-Gorj, 1964).

Gastropacha quercifolia ([Denis \& Schiffermüller], 1775): Pasărea, Pustnicu, 21.VII.-6.VIII.1996-2010, rare. Further records: Bucureşti, Comana (Popescu-Gorj, 1964).

Gastropacha populifolia ([Denis \& Schiffermüller], 1775): Brăneşti, 3.VIII.2004, 1 §. Further records: București, 17.VIII.1905, coll. Salay (Popescu-Gorj, 1964).

Odonestis pruni (Linnaeus, 1758): Brăneşti, Pasărea, Pustnicu, 25.VII.-8.VIII.19962009, common. Further records: Bucureşti (Popescu-Gorj, 1964).

\section{Family Endromidae}

Endromis versicolora (Linnaeus, 1758): Buftea, 5.IV.1890, coll. Salay (PopescuGorj, 1964).

\section{Family Saturniidae}

Aglia tau (Linnaeus, 1758)*: Cernica, Comana, Pasărea, 1-7.V.1997-98, rare. New record for the Romanian Plain.

Saturnia pyri ([Denis \& Schiffermüller], 1775): Brăneşti, Pasărea, Comana, 2-23.V.1999-2001, rare. Further records: Bucureşti (Popescu-Gorj, 1964).

Saturnia spini ([Denis \& Schiffermüller], 1775)**: Bucureşti, Comana, Filaret, 18951929 (Popescu-Gorj, 1964). Acceptable historical record, the species being reported from the northern territories of Bulgaria (Ruse, Razgrad, Pleven, Veliko Târnovo) (S. Beshkov, pers. comm.).

Perisomena caecigena (Kupido, 1825) (Fig. 2 B): Comana, 4.X.1915 (Popescu-Gorj, 1964), Bucureşti, 3.X.1934, leg. Niculescu (Nemeş \& Voicu, 1973), Cernica, 10-15. IX.1953, 23.IX.1954, ex. larva, leg. Niculescu (König, 1975), Comana, 15.IX.1949 (leg. A. Popescu-Gorj). Acceptable historical record, the species being recorded from the northern territories of Bulgaria (S. Beshkov, pers. comm.). 

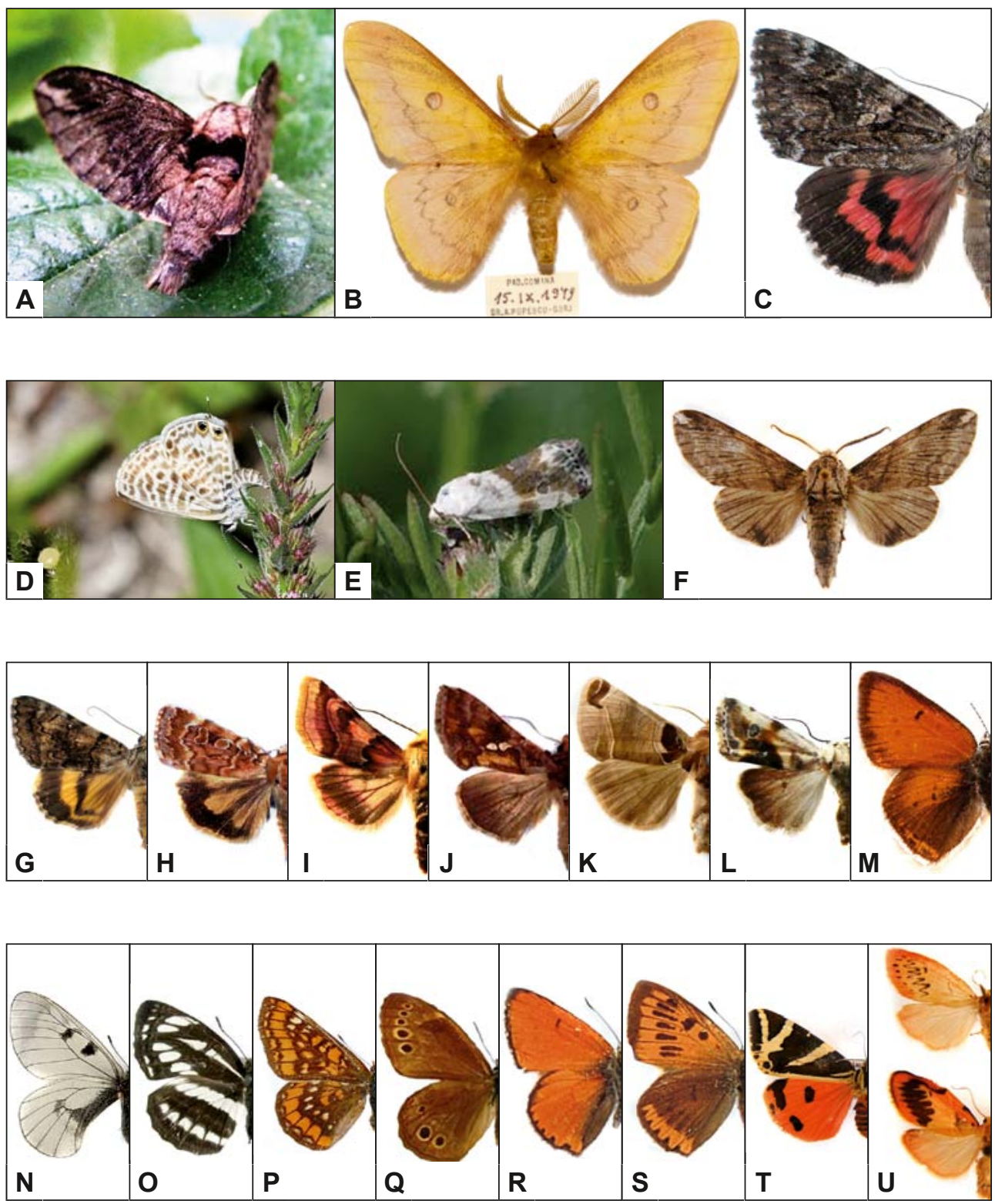

Fig. 2 - Dolbina elegans (A, F); Perisomena caecigena (B); Leptotes pirithous (D); Tarachidia candefacta (E, L); Catocala dilecta (C); Catocala nymphagoga (G); Noctua haywardi (H); Periphanes delphini (I); Chrysodeixis chalcites (J); Plusidia cheiranthi (K); Lycaena hippothoe (M); Parnassius mnemosyne $(\mathrm{N})$; Neptis sappho $(\mathrm{O})$; Euphydryas maturna $(\mathrm{P})$; Lopinga achine $(\mathrm{Q})$; Lycaena dispar (R, male; S, female); Euplagia quadripunctaria (T); Miltochrista miniata (U; bottom side - f.fasciata). Taxa protected by law at European or national level: A, F, K, M, N, O, P, Q, R, S, T. Foto: Levente Székely (A, C, F, G, H, I, J, K, L, N, O, P, Q, R, S, T, U); Iorgu Ionuţ Ştefan (B, M); Cosmin Ovidiu Manci (D); Cristian Mihai (E). 
Family Sphingidae

Marumba quercus ([Denis \& Schiffermüller], 1775): Brăneşti, Comana, Mihai Bravu, Pasărea, 12.VII.-1.VIII.1996-2004, very common. Further records: Bucureşti, Comana, Ciocăneşti (Popescu-Gorj, 1964).

Mimas tiliae (Linnaeus, 1758): Pasărea, Comana, Brăneşti, Pustnicu, 1-24.V. and 25.VII.-17.VIII.1996-2010, very common. Further records: Brăneşti, Cozieni (Popescu-Gorj, 1964).

Smerinthus ocellata (Linnaeus, 1758)*: Brănești, Pasărea, 21.VII.-2.VIII.1996-2001, rare.

Laothoe populi (Linnaeus, 1758): Brăneşti, Pasărea, Cernica, 30.V.-9.VIII.1996-2010, rare. Further records: Bucureşti (Popescu-Gorj, 1964).

Agrius convolvuli (Linnaeus, 1758): Brăneşti, Pasărea, Cernica, 23.VII.2.X.1997-2001, rare. Further records: Bucureşti, Otopeni (Popescu-Gorj,1964).

Sphinx ligustri (Linnaeus, 1758): Bucureşti (Popescu-Gorj, 1964).

Acherontia atropos (Linnaeus, 1758): București, Jilava, Brănești (Popescu-Gorj, 1964).

Dolbina elegans A. Bang-Haas, 1912 (Fig. 2 A, F): Brăneşti, Cernica, Cozieni, Păd. Buda, Pasărea, Pustnicu, Jilava, 7-23.V. and 24.VII.-7.VIII.1997-2010, in two broods, local common. Further records: Halta Cozieni (Popescu-Gorj, 1971). Distributed from SW-Ukraine and eastern Balkans to western and southern Turkey, northern Syria, western Iordan, northern Iraq and northern Iran. Not a very active species, with adults flying only for a short period after nightfall, usually quite close to the ground. The first specimens from Romania were taken in 1968 from Cozieni railway station near Brăneşti (Popescu-Gorj, 1971). Based on these specimens the subspecies steffensi Popescu-Gorj, 1971 has been described. There are no constant differences between the genitalia and morphology of European, Turkish and Syrian examples. This subspecific status is not justified for the European population, previously referred to as subsp. steffensi (Pittaway, 2010).

Hemaris croatica (Esper, 1779)**: Bucureşti-Botanical Garden (Caradja, 1947).

Macroglossum stellatarum (Linnaeus, 1758): Brăneşti, Pasărea, Pustnicu, Comana, Jilava, 25.VII.-2.X.1996-2010, common. Further records: Mihai Bravu (Popescu, 1939), Bucureşti, Comana (Popescu-Gorj, 1964).

Daphnis nerii (Linnaeus, 1758): Bucureşti, 8.VI.1890, coll. Salay (Popescu-Gorj, 1964).

Hyles euphorbiae (Linnaeus, 1758): Pasărea, 1-2.VIII.2002, rare. Further records: Mihai Bravu (Popescu, 1939).

Hyles livornica (Esper, 1779): Brăneşti, Pasărea, 31.VII.-17.VIII.1997-99, common. Further records: Bucureşti (Popescu-Gorj, 1964).

Deilephila elpenor (Linnaeus, 1758): Brăneşti, Pasărea, Pustnicu, 23.V.-28.VII.19962010, rare. Further records: Bucureşti (Popescu-Gorj, 1964).

Deilephila porcellus (Linnaeus, 1758): Comana, Pasărea, Pustnicu, 7.V.-17.VIII.19962010, common. Further records: Bucureşti, Comana (Popescu-Gorj, 1964).

Hippotion celerio (Linnaeus, 1758): Bucureşti (Salay, 1910). 
Family Hesperiidae

Erynnis tages (Linnaeus, 1758): Pasărea, Brăneşti, 25.VII.-8.VIII.1997-2009, common. Further records: Mihai Bravu (Popescu, 1939), Comana (Popescu-Gorj, 1964).

Carcharodus alceae (Esper, 1780): Bucureşti, Chitila, Comana, Jilava (PopescuGorj, 1964).

Carcharodus floccifera (Zeller, 1847): Brăneşti, Bucureşti, Comana, Filaret (Popescu-Gorj, 1964).

Spialia orbifer (Hübner, 1823): Comana, 19.V.1896, coll. Salay (Popescu-Gorj, 1964). Pyrgus malvae (Linnaeus, 1758): Bucureşti, Chitila, Comana (Popescu-Gorj, 1964). Pyrgus serratulae (Rambur, 1839)**: Buftea, Mogoşoaia (Popescu-Gorj, 1964). The species being recorded from the northern territories of Bulgaria, reported from Svishtov (Buresch \& Tuleschkow, 1930).

Pyrgus alveus (Hübner, 1803)**: Bucureşti, Comana (Popescu-Gorj, 1964).

Thymelicus lineola (Ochsenheimer, 1808): Brăneşti, Pasărea, 3.V.-25.VII.1996-99, rare. Further records: Comana (Popescu-Gorj, 1964).

Hesperia comma (Linnaeus, 1758): Brăneşti, Pasărea, 26-27.V.2001, rare. Further records: Mihai Bravu (Popescu, 1939); Bucureşti, Brăneşti, Comana, Filaret (Popescu-Gorj, 1964).

Ochlodes sylvanus (Esper, 1777): Brăneşti, Pasărea, 26-27.V.2001, rare. Further records: Bucureşti, Comana, Filaret, Ţigăneşti (Popescu-Gorj, 1964).

Family Papilionidae

Zerynthia polyxena ([Denis \& Schiffermüller], 1775)**: Bucureşti, 1892, leg. Joannis (Montandon, 1900; Niculescu, 1960); Comana, 15-20.IV.1911-1915, Filaret, 1.V.1905, Mihai Bravu, 1915 (Popescu-Gorj, 1964).

Parnassius mnemosyne distincta Bryk \& Eisner, 1930 (Fig. 2 N): Brăneşti, Pasărea, 1-14.V.2001-2008, very common. Further records: Bucureşti, Chitila, Comana, Țigăneşti (Montandon, 1900; Popescu-Gorj, 1964); Comana (König, 1975); Cernica (Niculescu, 1960).

Iphiclides podalirius (Linnaeus, 1758): Brăneşti, Cernica, Pasărea, Pustnicu, 1.V.-18. VIII.1996-2010, common. Further records: Mihai Bravu (Popescu, 1939); DudeştiCioplea (Niculescu, 1960); Băneasa, Bucureşti, Chitila, Păd. Andronache (Niculescu, 1960); Bucureşti, Brăneşti, Comana, Chitila, Filaret, Ţigăneşti (Popescu-Gorj, 1964). Papilio machaon (Linnaeus, 1758): Pasărea, Bucureşti, 25.VII.-8.VIII.1996-2007, rare. Further records: Bucureşti, Comana, Chitila (Popescu-Gorj, 1964).

\section{Family Pieridae}

Leptidea sinapis (Linnaeus, 1758): Brăneşti, Comana, Pasărea, 1-27.V.; 24.VI.-8. VIII.1996-2010, common. Further records: Mihai Bravu (Popescu, 1939); Păd. Andronache (Popescu-Gorj, 1964); Bucureşti, Cernica (Niculescu, 1963); Brăneşti, Bucureşti, Chitila, Comana, Ţigăneşti (Popescu-Gorj, 1964). 
Anthocharis cardamines (Linnaeus, 1758): Brăneşti, Pasărea, 1-26.V.2001-2003, rare. Further records: Bucureşti, Cernica, Comana, Budeşti (Niculescu, 1963); Brăneşti, Bucureşti, Comana, Chitila, Ţigăneşti (Popescu-Gorj, 1964).

Aporia crataegi (Linnaeus, 1758): Brăneşti, Pasărea, 26-27.V.2001, common. Further records: Bucureşti, Cernica (Niculescu, 1963); Comana, Chitila, Filaret (PopescuGorj, 1964).

Pieris brassicae (Linnaeus, 1758): Pasărea, 2.V.-17.VIII.1996-2010, common. Further records: Mihai Bravu (Popescu, 1939); Bucureşti, Dudeşti-Cioplea (Niculescu, 1963); Comana, Chitila (Popescu-Gorj, 1964).

Pieris mannii (Meyer, 1851): Giurgiu (Popescu-Gorj, 1960; Niculescu, 1963). Acceptable historical record, the species being recorded from the northern territories of Bulgaria (Lovech and Târnovo) (S. Beshkov, pers. comm.).

Pieris rapae (Linnaeus, 1758): Pasărea, Brăneşti, 2.V-3.X.1996-2010, common. Further records: Mihai Bravu (Popescu, 1939); Bucureşti, Cernica, Dudeşti-Cioplea (Niculescu, 1963); Chitila, Jilava (Popescu-Gorj, 1964).

Pieris balcana Lorkovic, 1970*: Pasărea, 2-4.V.2002, rare. It is a poorly known species in Romania with few records scattered across the country's territory. New record for the Romanian Plain.

Pieris napi (Linnaeus, 1758): Pasărea, Brăneşti, 2.V.-3.X.1996-2010, common. Further records: Mihai Bravu (Popescu, 1939); Bucureşti, (Niculescu, 1963); Brăneşti, Chitila, Comana, Jilava, Ţigăneşti (Popescu-Gorj, 1964).

Pontia edusa (Fabricius, 1777): Pasărea, Cernica, 24.VII.-17.X.1996-2010, common. Further records: Mihai Bravu (Popescu, 1939); Bucureşti (Niculescu, 1963); Brăneşti, Chitila, Comana, Jilava, Ţigăneşti (Popescu-Gorj, 1964).

Colias erate (Esper, 1805): Pasărea, Pustnicu, 26.V.-20.X.1996-2009, common. Further records: Dudeşti-Cioplea (Niculescu, 1963); Bucureşti, Comana, Mogoşoaia, Pasărea, Ştefăneşti (Popescu-Gorj, 1978).

Colias croceus (Fourcroy, 1785): Pasărea, 1.VIII.-3.X.1996-2010, rare. Further records: Mihai Bravu (Popescu, 1939); Bucureşti (Niculescu, 1963); Brăneşti, Chitila, Comana, Jilava, Ţigăneşti (Popescu-Gorj, 1964).

Colias chrysotheme (Esper, 1781)**: Păd. Andronache (Popescu-Gorj, 1938); Dudeşti-Cioplea (Niculescu, 1963).

Colias hyale (Linnaeus, 1758): Cernica, Dudeşti-Cioplea (Niculescu, 1963); Bucureşti, Chitila, Comana, Filaret, Jilava, Țigăneşti (Popescu-Gorj, 1964).

Colias alfacariensis Ribbe, 1905*: Pasărea, 27.V.2001, 1 స. New record for the Romanian Plain.

Gonepteryx rhamni (Linnaeus, 1758): Bucureşti, Cernica (Niculescu, 1963); Bucureşti, Chitila, Comana (Popescu-Gorj, 1964). 
Family Riodinidae

Hamearis lucina (Linnaeus, 1758): Pasărea, 2-4.V.2002, common. Further records: Bucureşti, Brăneşti, Chitila, Ţigăneşti (Popescu-Gorj, 1964).

Family Lycaenidae

Lycaena phlaeas (Linnaeus, 1761): Pasărea, 6.X.2001, 1 ․ Further records: București, Brăneşti, Comana, Ţigăneşti (Popescu-Gorj, 1964).

Lycaena helle ([Denis \& Schiffermüller], 1775)**: Bucureşti, Chitila, 20.V.1889, coll. Salay (Popescu-Gorj, 1964).

Lycaena dispar rutila (Werneburg, 1864) (Fig. 2 R, S): Brăneşti, Pasărea, 2-4.V.2002, rare. Further records: Bucureşti, Chitila, Comana, Jilava, Țigăneşti (Popescu-Gorj, 1964).

Lycaena virgaureae (Linnaeus, 1758): Mihai Bravu (Popescu, 1939); Comana, 15.VII.1916 (Popescu-Gorj, 1964).

Lycaena tityrus (Poda, 1761): Comana, 26.VI.2010, rare, leg. C. Mihai. Further records: Mihai Bravu (Popescu, 1939); Buftea, Ţigăneşti (Popescu-Gorj, 1964).

Lycaena hippothoe (Linnaeus, 1758)** (Fig. 2 M): Bucureşti, Chitila, 8.VI.1889, coll. Salay (Popescu-Gorj, 1964). Acceptable historical record, the species being recorded from the northern territories of Bulgaria (Markowitsch, 1904, 1909; Drenowski, 1907, 1930; Buresch \& Tuleschkow, 1930).

Lycaena thersamon (Esper, 1784): Comana, 27.VI.2010, rare, leg. C. Mihai. Further records: Mihai Bravu (Popescu, 1939); Bucureşti, Brăneşti, Chitila, Comana, Jilava, Ţigăneşti (Popescu-Gorj, 1964).

Thecla betulae (Linnaeus, 1758): Buftea, Comana, Ţigăneşti (Popescu-Gorj, 1964). Neozephyrus quercus (Linnaeus, 1758): Comana, Cernica, Pasărea, 12.VII.-8. VIII.1997-99, very common. Further records: Brăneşti, Ţigăneşti (Popescu-Gorj, 1964).

Calophrys rubi (Linnaeus, 1758): Pasărea, 2-4.V.2002, common. Further records: Bucureşti, Comana, Filaret, Ţigăneşti (Popescu-Gorj, 1964).

Satyrium pruni (Linnaeus, 1758)*: Pasărea, 26-27.V.2001, very common.

Satyrium spini ([Denis \& Schiffermüller], 1775): Chitila, Comana, Țigăneşti (Popescu-Gorj, 1964).

Satyrium ilicis (Esper, 1779): Comana, Ţigăneşti (Popescu-Gorj, 1964).

Leptotes pirithous (Linnaeus, 1758)* (Fig. 2 D): Comana, 27.VIII.2009, 1 +, leg. C. O. Manci. New record for the Romanian Plain.

Everes argiades (Pallas, 1771): Comana, 10-11.VII.2010, rare, leg. C. Mihai. Further records: Bucureşti, Brăneşti, Chitila, Jilava (Popescu-Gorj, 1964).

Everes alcetas (Hoffmannsegg, 1804): Comana, 10-11.VII.2010, rare, leg. C. Mihai. Further records: Bucureşti, Comana, Jilava (Popescu-Gorj, 1964). It is a poorly known species in Romania with few records scattered across the country's territory. Cupido minimus (Fuessly, 1775)*: Pustnicu, 8.V.2010, 1 ठे.

Celastrina argiolus (Linnaeus, 1758): Brăneşti, Pasărea, 25.VII.-9.VIII.1997-2010, rare. Further records: Bucureşti, Chitila, Comana, Jilava, Ţigăneşti (Popescu-Gorj, 1964). 
Glaucopsyche alexis (Poda, 1761): Comana (Popescu-Gorj, 1964).

Maculinea arion (Linnaeus, 1758)**: Brăneşti (Popescu-Gorj, 1964).

Plebejus argus (Linnaeus, 1761): Brăneşti, Pasărea, 7.V.-6.VIII.1996-2010, rare. Further records: Mihai Bravu (Popescu, 1939); Bucureşti, Brăneşti, Filaret, Chitila, Comana, Jilava (Popescu-Gorj, 1964).

Plebejus argyrognomon (Bergstässer, 1779): Bucureşti, Chitila, Ţigăneşti (PopescuGorj, 1964).

Aricia agestis ([Denis \& Schiffermüller], 1775): Brăneşti, Pasărea, Cernica, 2.V.-25. VII.1996-2009, rare. Further records: Bucureşti, Chitila, Comana (Popescu-Gorj, 1964).

Cyaniris semiargus (Rottemburg, 1775): Brăneşti, Comana, Ţigăneşti (PopescuGorj, 1964).

Polyommatus icarus (Rottemburg, 1775): Pasărea, Brăneşti, 1.V.-2.X.1996-2010, very common. Further records: Mihai Bravu (Popescu, 1939); Bucureşti, Brăneşti, Comana, Filaret, Ţigăneşti (Popescu-Gorj, 1964).

Polyommatus thersites (Cantener, 1834)*: Brăneşti, Cernica, 2.V.-25.VII.1996-99, very rare.

Meleageria bellargus (Rottemburg, 1775): Pasărea, 27.V.2001, rare. Further records: Bucureşti, Brăneşti, Comana, Filaret, Jilava (Popescu-Gorj, 1964).

Meleageria coridon (Poda, 1761): Bucureşti, Filaret (Popescu-Gorj, 1964).

Family Nymphalidae

Subfamily Nymphalinae

Libythea celtis (Laicharting, 1782): Comana, 15.VI.2010. (Obs. S. M. Stanciu). Requiring reconfirmation!

Argynnis paphia (Linnaeus, 1758): Brăneşti, Pasărea, Pustnicu, 25.VII.-9.VIII.19962010, common. Further records: Mihai Bravu (Popescu, 1939); Cernica (Niculescu, 1965); Bucureşti, Chitila, Ţigăneşti (Popescu-Gorj, 1964).

Argynnis pandora ([Denis \& Schiffermüller], 1775): Brăneşti, Pasărea, Cernica, 26.V.-23.VIII.1996-2010, rare. Further records: Mihai Bravu (Popescu, 1939); Cernica (Niculescu, 1965); Bucureşti, Comana (Popescu-Gorj, 1964).

Argynnis aglaja (Linnaeus, 1758): Mihai Bravu (Popescu, 1939); Cernica, Ştefăneşti (Niculescu, 1965); Comana, Chitila (Popescu-Gorj, 1964).

Argynnis adippe ([Denis \& Schiffermüller], 1775): Mihai Bravu (Popescu, 1939); Băneasa, Cernica, Ştefăneşti (Niculescu, 1965); Comana, Chitila (Popescu-Gorj, 1964).

Argynnis niobe (Linnaeus, 1758): Cernica, Comana (Niculescu, 1965).

Issoria lathonia (Linnaeus, 1758): Pasărea, Pustnicu, 25.VII.-3.X.1996-2010, very common. Further records: Mihai Bravu (Popescu, 1939); Cernica (Niculescu, 1965); Bucureşti, Chitila, Comana, Mihai Bravu (Popescu, 1939); Cernica, Ştefăneşti (Niculescu, 1965); Comana, Chitila (Popescu-Gorj, 1964). 
Brenthis daphne ([Denis \& Schiffermüller], 1775): Comana, 27.VI.2010, rare, leg. C. Mihai. Further records: Comana (Caradja, 1896; Bucureşti, 5.VII.1915, Comana, 4.VI.1911 (Popescu-Gorj, 1964); Cernica (Niculescu, 1965).

Brenthis hecate ([Denis \& Schiffermüller], 1775)**: Ţigăneşti, 4.VI.1916 (PopescuGorj, 1964). Acceptable historical record, the species being recorded from the northern territories of Bulgaria (Lovech, Razgrad, Samuil) (S. Beshkov, pers. comm.).

Clossiana euphrosyne (Linnaeus, 1758): Bucureşti, Chitila, Comana, Ţigăneşti (Popescu-Gorj, 1964); Băneasa, Cernica (Niculescu, 1965).

Clossiana selene ([Denis \& Schiffermüller], 1775): Comana, Ţigăneşti (PopescuGorj, 1964); Comana (Niculescu, 1965).

Clossiana dia (Linnaeus, 1767): Pasărea, 2-4.V.2002, common. Further records: Bucureşti, Comana, Jilava (Popescu-Gorj, 1964); Buftea, Chitila, Cernica, Păd. Andronache (Niculescu, 1965).

Vanessa atalanta (Linnaeus, 1758): Pasărea, 25.VII.-9.VIII.1996-2009, rare. Further records: Mihai Bravu (Popescu, 1939); Bucureşti, Comana (Popescu-Gorj, 1964); Cernica (Niculescu, 1965).

Vanessa cardui (Linnaeus, 1758): Brăneşti, Pasărea, 24.VII.-3.X.1996-2010, common. Further records: Mihai Bravu (Popescu, 1939); Bucureşti, Chitila, Comana, Jilava (Popescu-Gorj, 1964); Comana, Dudeşti-Cioplea (Niculescu, 1965).

Inachis io (Linnaeus, 1758): Pasărea, 25.VII.-9.VIII.1996-2010, rare. Further records: Comana (Popescu-Gorj, 1964); Bucureşti (Niculescu, 1965).

Aglais urticae (Linnaeus, 1758): Pasărea, 25.VII.1997, rare. Further records: Comana (Popescu-Gorj, 1964).

Polygonia c-album (Linnaeus, 1758): Pasărea, Pustnicu, 4.V.-3.X.1996-2010, common. Further records: Mihai Bravu (Popescu, 1939); Bucureşti (Niculescu, 1965).

Polygonia egea (Cramer, 1775)**: Bucureşti, leg. A. L. Montadon (De Joannis, 1892; Niculescu, 1965). Acceptable historical record, the species being recorded from the northern territories of Bulgaria: Resseletz near Tcherven Bryag, the most northern locality in Bulgaria (S. Beshkov, pers. comm.).

Araschnia levana (Linnaeus, 1758): Brăneşti, 2-4.V.2002, common. Further records: Comana, Ţigăneşti (Popescu-Gorj, 1964; Niculescu, 1965).

Nymphalis antiopa (Linnaeus, 1758): Bucureşti, Comana, Cernica, Buftea, Ţigăneşti (Popescu-Gorj, 1960, 1964).

Nymphalis polychloros (Linnaeus, 1758): Pasărea, 22.VII.1997, 1 q. Further records: Mihai Bravu (Popescu, 1939); Bucureşti, Comana (Popescu-Gorj, 1964).

Nymphalis xanthomelas ([Denis \& Schiffermüller], 1775): Păd. Andronache, 1936, leg. Popescu-Gorj (Niculescu, 1965).

Nymphalis vaualbum ([Denis \& Schiffermüller], 1775)**: Comana, 7.VIII.1915 (Popescu-Gorj, 1964). 
Euphydryas maturna (Linnaeus, 1758) (Fig. 2 P): Brăneşti, Pasărea, 26-27.V.2001; 26.V.2008 (leg. Stanciu \& Görbe), very common. Further records: Bucureşti, Brăneşti, Chitila, Ţigăneşti (Popescu-Gorj, 1964); Afumaţi, Băneasa, Cernica, Ştefăneşti (Niculescu, 1965).

Melitaea cinxia (Linnaeus, 1758): Bucureşti, Comana, Filaret, Ţigăneşti (PopescuGorj, 1964); Cernica (Niculescu, 1965).

Melitaea phoebe ([Denis \& Schiffermüller], 1775): Pasărea, 26.V.-25.VII.1997-2009, rare. Further records: Bucureşti, Brăneşti, Comana, Ţigăneşti (Popescu-Gorj, 1964); Cernica (Niculescu, 1965).

Melitaea ornata ogygia Frusthorfer, 1908*: Comana, 11.VII.2010, very rare, leg. C. Mihai. Requiring reconfirmation!

Melitaea aurelia Nickerl, 1850: Bucureşti, coll. Salay (Popescu-Gorj, 1964).

Melitaea trivia ([Denis \& Schiffermüller], 1775) : Comana, Brăneşti, 26.V.-10. VII.1997-99, very rare. Further records: Băneasa, Cernica, Ştefăneşti, Păd. Andronache, Pasărea (Niculescu, 1965).

Melitaea didyma ([Denis \& Schiffermüller], 1775): Comana, 10-11.VII.2010, rare, leg. C. Mihai. Further records: Brăneşti, Comana, 7.VII.-2.VIII.1916 (Popescu-Gorj, 1964); Bucureşti, Cernica (Niculescu, 1965).

Melitaea arduinna (Esper, 1784)**: Bucureşti (Caradja, 1895; Fleck, 1900; Niculescu, 1965). Acceptable historical record, the species being recorded from the northern territories of Bulgaria: Sboryanovo (Buresch \& Tuleschkow, 1929, 1930; Abadjiev, 1995).

Melitaea athalia (Rottemburg, 1775): Mihai Bravu (Popescu, 1939); Brăneşti, Comana, Ţigăneşti (Popescu-Gorj, 1964); Cernica, Ştefăneşti (Niculescu, 1965).

Limenitis populi (Linnaeus, 1758)**: Comana, 1916 (Niculescu, 1965).

Neptis sappho (Pallas, 1771) (Fig. 2 O): Brăneşti, Pasărea, 2-4.V.2002, common. Further records: Băneasa, Bucureşti, Chitila, Comana, Ţigăneşti (Popescu-Gorj, 1964); Bucureşti (Niculescu, 1965).

Neptis rivularis (Scopoli, 1763): București, Chitila, Comana (Popescu-Gorj, 1964; Niculescu, 1965).

Apatura metis Freyer, 1829: Brăneşti, 3-4.V.2002, rare, Comana, 29.V.2009, rare. Further records: Giurgiu (Popescu-Gorj, 1960; Niculescu, 1965); Budeşti-Bucureşti (Niculescu, 1965).

Apatura ilia ([Denis \& Schiffermüller], 1775): Bucureşti (Popescu-Gorj, 1964). Apatura iris (Linnaeus, 1758)**: Bucureşti, 27.VII.1919 (Popescu-Gorj, 1964).

Family Nymphalidae

Subfamily Satyrinae

Kirinia roxelana (Cramer, 1777)**: Comana, 20.VI.1916 (Popescu-Gorj, 1964).

Pararge aegeria tircis Butler, 1867: Mihai Bravu (Popescu, 1939); Bucureşti, Brăneşti, Chitila, Comana, Filaret, Ţigăneşti (Popescu-Gorj, 1964).

Lasiommata megera (Linnaeus, 1758): Bucureşti, Chitila, Comana, Filaret, Ţigăneşti (Popescu-Gorj, 1964). 
Lasiommata maera (Linnaeus, 1758): Pasărea, 3-4.V.2002, rare. Further records: Bucureşti, Brăneşti, Chitila, Comana, Țigăneşti (Popescu-Gorj, 1964).

Lopinga achine (Scopoli, 1763) (Fig. 2 Q): Comana, Romăneşti, 6.VI.-12.VII.19972004, rare. Further records: Bucureşti, Chitila, Comana, Dudeşti-Cioplea, Ţigăneşti (Popescu-Gorj, 1964).

Coenonympha arcania (Linnaeus, 1761): Brăneşti, Ţigăneşti (Popescu-Gorj, 1964). Coenonympha glycerion (Borkhausen, 1788): București, Brăneşti, Chitila, Comana (Popescu-Gorj, 1964).

Coenonympha pamphilus (Linnaeus, 1758): Pasărea, Cernica, Pustnicu, 24.VII.2.X.1996-2010, common. Further records: Bucureşti, Brăneşti, Chitila, Comana, Filaret, Jilava, Ţigăneşti (Popescu-Gorj, 1964).

Aphanthopus hyperanthus (Linnaeus, 1758): Comana, Brăneşti, Chitila, 1892-1916 (Popescu-Gorj, 1964).

Maniola jurtina (Linnaeus, 1758): Pasărea, 24.VII.-9.VIII.1996-2010, common. Further records: Mihai Bravu (Popescu, 1939); Bucureşti, Brăneşti, Comana, Chitila, Ţigăneşti (Popescu-Gorj, 1964).

Hyponephele lycaon (Rottemburg, 1775)**: Bucureşti, Chitila, 1890-1916, coll. Salay (Popescu-Gorj, 1964).

Hyponephele lupina (O. G. Costa, 1836)**: Brăneşti, 20.VIII.1915, coll. Salay (Popescu-Gorj, 1977).

Erebia ligea (Linnaeus, 1758)**: Chitila, 26.VI.1889, coll. Salay (Popescu-Gorj, 1964).

Melanargia galathea (Linnaeus, 1758): Comana, 12-13.VII.1996, common. Further records: Bucureşti, Brăneşti, Comana, Ţigăneşti (Popescu-Gorj, 1964).

Minois dryas (Scopoli, 1763): Pasărea, Comana, 10.VII.-17.VIII.1999-2010, common. Further records: Mihai Bravu (Popescu, 1939); Băneasa, Bucureşti, Brăneşti, Chitila, Comana (Popescu-Gorj, 1964).

Hipparchia fagi (Scopoli, 1763): Comana, 5.VIII.1890, coll. Salay (Popescu-Gorj, 1964).

Hipparchia semele (Linnaeus, 1758): Comana, 1915-1916 (Popescu-Gorj, 1964) Arethusana arethusa ([Denis \& Schiffermüller], 1775): Brăneşti, Comana, Ţigăneşti, 1886-1927 (Popescu-Gorj, 1964).

Brinthesia circe (Fabricius, 1775): Comana, 1893-1927 (Popescu-Gorj, 1964).

Family Drepanidae

Thyatira batis (Linnaeus, 1758): Brăneşti, Pasărea, Pustnicu, 21.VII.-9.VIII.19962010, very common. Further records: Bucureşti (Popescu-Gorj, 1964).

Habrosyne pyritoides (Hufnagel, 1766)*: Brăneşti, Comana, Pasărea, 22.V.-9. VIII.1996-2010, very common.

Tethea ocularis (Linnaeus, 1758): Brănești, Pasărea, 30-31.V.2001-2002, rare. Further records: Bucureşti (Popescu-Gorj, 1964). 
Watsonalla binaria (Hufnagel, 1767): Brăneşti, Pasărea, Pustnicu, 7.V.-8.VIII.19962010, common. Further records: București, Filaret (Popescu-Gorj, 1964).

Drepana falcataria (Linnaeus, 1758): București, Comana (Montandon, 1900; Popescu-Gorj, 1964).

Cilix glaucatus (Scopoli, 1763): Brănești, Pasărea, 1-9.VIII.1997-2001, rare. Further records: Bucureşti, Chitila (Popescu-Gorj, 1964).

\section{Family Geometridae}

Abraxas grossulariata (Linnaeus, 1758): Pasărea, 30.V.1998, 1 §. Further records: Bucureşti, Chitila, Comana (Popescu-Gorj, 1964).

Lomaspilis marginata (Linnaeus, 1758): Comana, Pasărea, Pustnicu, 21.V.-28. VII.1996-2010, common. Further records: Bucureşti, Comana, Filaret (PopescuGorj, 1964).

Ligdia adustata ([Denis \& Schiffermüller], 1775): Brăneşti, Pasărea, Pustnicu, 21.V.9.VIII.1996-2010, rare. Further records: Bucureşti, Chitila, Ţigăneşti (Popescu-Gorj, 1964).

Stegania dilectaria (Hübner, 1790): Brăneşti, Pasărea, 3-4.V.2002, rare. Further records: Bucureşti, 22.V.1904, coll. Salay (Popescu-Gorj, 1964).

Heliommata glarearia ([Denis \& Schiffermüller], 1775): Pasărea, Pustnicu, 28.VII.8.VIII.1996-2010, common. Further records: București, Comana, Chitila (PopescuGorj, 1964).

Macaria notata (Linnaeus, 1758)*: Pasărea, Pustnicu, 21.V.-21.VII.1996-2009, common.

Macaria alternata ([Denis \& Schiffermüller], 1775): Bucureşti (Popescu-Gorj, 1964).

Chiasmia clathrata (Linnaeus, 1758): Brăneşti, Pasărea, Cernica, Pustnicu, 30.V.9.VIII.1996-2010, common. Further records: Bucureşti, Brăneşti, Chitila, Jilava (Popescu-Gorj, 1964).

Godonella aestimaria sareptanaria (Staudinger, 1891): Bucureşti (Popescu-Gorj, 1964).

Tephrina murinaria ([Denis \& Schiffermüller], 1775): Pasărea, Cernica, 30.V.-21. VII.1996-2009, rare. Further records: Bucureşti, Comana, Filaret (Popescu-Gorj, 1964).

Tephrina arenacearia ([Denis \& Schiffermüller], 1775): Brăneşti, Pasărea,1531.V.1996-2010, common. Further records: Mihai Bravu (Popescu, 1939); Bucureşti, Comana, Jilava (Popescu-Gorj, 1964).

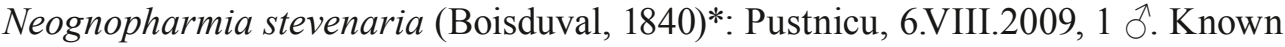
only from the South and the East of Romania (the Extra-Carpathian areas), from Banat (Orşova, Băile Herculane), Moldova (Iaşi, Tecuci, Hanul Conachi), Muntenia (Amara, Slobozia). It is a common species in Dobrogea and in the Danube Delta. Petrophora chlorosata (Scopoli, 1763)*: Pasărea, 26.V.2001, rare. 
Plagodis pulveraria (Linnaeus, 1758)*: Comana, Cernica, Pasărea, Pustnicu, 21.VII.1.VIII.1996-2010, very common.

Plagodis dolabraria (Linnaeus, 1758): Comana, 12.V.1919 (Popescu-Gorj, 1964).

Opistograpthis luteolata (Linnaeus, 1758): Comana (Popescu-Gorj, 1964).

Therapis flavicaria ([Denis \& Schiffermüller], 1775): Brănești, Pasărea, 30.V.-25. VII.1996-2009, rare. Further records: București, Chitila, Comana, Filaret (PopescuGorj, 1964).

Pseudopanthera macularia (Linnaeus, 1758): Comana, Pasărea, Cernica, 16.V.-8. VIII.1996-2010, common. Further records: Bucureşti, Chitila, Comana, Filaret, Ţigăneşti (Popescu-Gorj, 1964).

Eilicrinia cordiaria (Hübner, 1790): Pasărea, 7-8.VIII.1997, rare. Further records: Bucureşti (Popescu-Gorj, 1964).

Eilicrinia trinotata (Metzner, 1845): Bucureşti (Popescu-Gorj, 1964); Păd. Andronache, 28.V.1936 (König, 1975).

Apeira syringaria (Linnaeus, 1758)*: Brănești, Pasărea, Cernica, 30.V.-15.IX.19962009, rare. New record for the Romanian Plain.

Ennomos autumnaria (Werneburg, 1859): Comana (Popescu-Gorj, 1964).

Ennomos quercinaria (Hufnagel, 1767): Buftea, Comana, 1890, coll. Salay (PopescuGorj, 1964).

Ennomos fuscantaria (Haworth, 1809): Buftea, Bucureşti (Popescu-Gorj, 1964).

Ennomos erosaria ([Denis \& Schiffermüller], 1775): Comana, 18.VII.1915 (PopescuGorj, 1964).

Selenia dentaria (Fabricius, 1775)*: Comana, Pasărea, 12-31.VII.1996-2010, common.

Selenia lunularia (Hübner, 1788): Pasărea, Pustnicu, 28.VII.-8.VIII.1996-97, rare. Further records: Bucureşti, Comana (Popescu-Gorj, 1964).

Selenia tetralunaria (Hufnagel, 1767)*: Pasărea, 25.VII.1997, 1 ․ New record for the Romanian Plain.

Artiora evonymaria ([Denis \& Schiffermüller], 1775): Pasărea, 6.X.2001, 1 q. Further records: Bucureşti, Chitila (Popescu-Gorj, 1964).

Crocallis tusciaria ([Denis \& Schiffermüller], 1775): Pasărea, 6-20.X.2001, rare. Further records: Bucureşti, 11.X.1910, coll. Salay (Popescu-Gorj, 1964).

Crocallis elinguaria (Linnaeus, 1758)*: Cernica, Pasărea, Pustnicu, 24-31.VII.19962009, common. New record for the Romanian Plain.

Colotois pennaria (Linnaeus, 1758)*: Bucureşti, 27.XI.2001, 1 ઈ.

Lycia hirtaria (Clerck, 1759): Bucureşti (Popescu-Gorj, 1964).

Briston betularia (Linnaeus, 1758)*: Brăneşti, Comana, Pasărea, Pustnicu, 12.VII.8.VIII.1996-2009, common.

Agriopis marginaria (Fabricius, 1766): București, Comana, Țigăneşti (PopescuGorj, 1964).

Erannis defoliaria (Clerck, 1759): Bucureşti, 30.XI.2001, 1 §. Further records: Bucureşti, Comana (Popescu-Gorj, 1964). 
Menophra abruptaria (Thunberg, 1792)**: Muntenia ? (Abafi-Aigner, 1901). Synopsia sociaria (Hübner, 1799)*: Pasărea, 25.VII.1997, very rare.

Peribatodes rhomboidaria ([Denis \& Schiffermüller], 1775): Pasărea, 30.V.-31. VII.1996-2009, rare. Further records: Bucureşti, Comana, Ţigăneşti (Popescu-Gorj, 1964).

Alcis repandata (Linnaeus, 1758)*: Pustnicu, 21.V.2009, common. New record for the Romanian Plain.

Hypomecis roboraria ([Denis \& Schiffermüller], 1775): Brăneşti, Comana, Cernica, Pasărea, Pustnicu, 21.V.-9.VIII.1996-2010, very common. Further records: Bucureşti, Comana (Popescu-Gorj, 1964).

Hypomecis punctinalis (Scopoli, 1763): București, Comana, Filaret, Otopeni (Popescu-Gorj, 1964).

Ascotis selenaria ([Denis \& Schiffermüller], 1775): Bucureşti, Comana, Pasărea, Pustnicu, 21.V.-9.VIII.1996-2010, very common. Further records: Bucureşti, Comana (Popescu-Gorj, 1964).

Ematurga atomaria (Linnaeus, 1758): Pasărea, Cernica, 21.VII.-8.VIII.1996-2010, very common. Further records: Bucureşti, Brăneşti, Chitila, Comana, Filaret, Ţigăneşti (Popescu-Gorj, 1964).

Cabera pusaria (Linnaeus, 1758): Pasărea, Pustnicu, 24.VII.-1.VIII.1996-2009, rare. Further records: Bucureşti, Chitila, Comana (Popescu-Gorj, 1964).

Cabera exanthemata (Scopoli, 1763): Pasărea, 25.VII.1997, very rare. Further records: Bucureşti, Chitila, Comana (Popescu-Gorj, 1964).

Siona lineata (Scopoli, 1763): Bucureşti, Comana, Filaret (Popescu-Gorj, 1964).

Brephos puella (Esper, 1787): Păd. Andronache (Popescu-Gorj, 1960).

Alsophila aceraria ([Denis \& Schiffermüller], 1775): Comana, 20.X.1915 (PopescuGorj, 1964).

Orthostixis cribraria (Hübner, 1759): Comana, 11-24.VI.1916 (Popescu-Gorj, 1964). Pseudoterpna pruinata (Hufnagel, 1767): Comana, 24.VI.1916 (Popescu-Gorj, 1964). Comibaena bajularia ([Denis \& Schiffermüller], 1775)*: Comana, Cernica, Pasărea, 30.V.-12.VII.1996-2008, very common. New record for the Romanian Plain.

Antonechloris smaragdaria (Fabricius, 1787): Pasărea, 8.VIII.1997, rare. Further records: Bucureşti, Chitila, Jilava (Popescu-Gorj, 1964).

Hemithaea aestivaria (Hübner, 1789): Comana, Țigăneşti (Popescu-Gorj, 1964).

Chlorissa viridata (Linnaeus, 1758): Bucureşti, Jilava (Popescu-Gorj, 1964).

Chlorissa cloraria (Hübner, 1813)*: Brăneşti, Pasărea, 25.VII.-2.VIII.1996-97, rare.

Thalera fimbrialis (Scopoli, 1763): Pasărea, 21.VII.-1.VIII.1996-97, rare. Further records: Bucureşti, Chitila (Popescu-Gorj, 1964).

Cyclophora albiocellaria (Hübner, 1789): Pasărea, 21.VII.1997, rare. Further records: Brăneşti, Comana (Popescu-Gorj, 1964).

Cyclophora annularia (Fabricius, 1775): Comana, Pasărea, Pustnicu, 21.V.-1. VIII.1996-2008, common. Further records: Comana (Popescu-Gorj, 1964). 
Cyclophora puppillaria (Hübner, 1799)*: Pasărea, 21.VII.1997, very rare. New record for the Romanian Plain.

Cyclophora porata (Linnaeus, 1767): Pasărea, 1.VIII.-15.IX.1996-2001, rare. Further records: Bucureşti, Brăneşti (Popescu-Gorj, 1964).

Cyclophora punctaria (Linnaeus, 1758): Pustnicu, 21.V.2009, common. Further records: Comana (Popescu-Gorj, 1964).

Cyclophora suppunctaria (Zeller, 1847): Pasărea, 21.VII.1997, 1 +, Pustnicu, 28.VII.2008, rare. Further records: Comana, 16.VII.1916 (Popescu-Gorj, 1964).

Timandra comae A. Schmidt, 1931: Brăneşti, Pasărea, Pustnicu, 21.VII.-9.VIII.19962010, very common. Further records: Bucureşti, Chitila, Jilava (Montandon, 1900; Popescu-Gorj, 1964).

Scopula tessellaria (Boisduval, 1840): Brăneşti, Comana (Popescu-Gorj, 1964).

Scopula nigropunctata (Hufnagel, 1767)*: Pasărea, 30.V.1998, rare. New record for the Romanian Plain.

Scopula virgulata ([Denis \& Schiffermüller], 1775): Comana (Popescu-Gorj, 1964). Scopula ornata (Scopoli, 1763): Bucureşti, Comana, Jilava (Popescu-Gorj, 1964).

Scopula rubiginata (Hufnagel, 1767): Bucureşti (Montandon, 1900), Comana, Pasărea, 12.VII.-16.IX., rare.

Scopula marginepunctata (Goeze, 1781): Mihai Bravu (Popescu, 1939); Bucureşti (Popescu-Gorj, 1964).

Scopula incanata (Linnaeus, 1758)*: Pasărea, 8.VIII.1997, 1 q, Pustnicu, 28.VII.2008, common.

Scopula immutata (Linnaeus, 1758): Comana, Păd. Călugăreni (Popescu-Gorj, 1964).

Scopula flaccidaria (Zeller, 1852): Giurgiu (Popescu-Gorj, 1960).

Scopula floslactata (Haworth, 1809)*: Comana, 12-13.VII.1997, very rare.

Idaea ochrata (Scopoli, 1763): Brăneşti, Comana (Popescu-Gorj, 1964).

Idaea muricata (Hufnagel, 1767): Brăneşti, Pasărea, Cernica, 30.V.-25.VII.1996-99, rare. Further records: Bucureşti, Otopeni (Popescu-Gorj, 1964).

Idaea rusticata ([Denis \& Schiffermüller], 1775)*: Pasărea, 1-8.VIII.1996-2008, rare.

Idaea biselata (Hufnagel, 1767): Comana (Popescu-Gorj, 1964).

Idaea inquinata (Scopoli, 1763): Bucureşti, Filaret (Popescu-Gorj, 1964); Comana (König, 1975).

Idaea politaria (Hübner, 1799)*: Pasărea, 21-25.VII.1996-98, very rare.

Idaea seriata (Schrank, 1802)*: Pasărea, 7-8.VIII.1997, very rare.

Idaea dimidiata (Hufnagel, 1767): Pasărea, 25.VIII.1997, very rare. Further records: Bucureşti (Popescu-Gorj, 1964).

Idaea camparia (Herrich-Schäffer, 1852): Bucureşti, 12.VIII.1941 (Popescu-Gorj, 1964).

Idaea pallidata ([Denis \& Schiffermüller], 1775):Comana, Ţigăneşti (Popescu-Gorj, 1964). 
Idaea aversata (Linnaeus, 1758): Brăneşti, Pasărea, Pustnicu, 30.V.-1.VIII.19962010, common. Further records: Bucureşti, Comana, (Popescu-Gorj, 1964).

Idaea deversaria (Herrich-Schäffer, 1848): Giurgiu (Popescu-Gorj, 1964).

Idaea degeneraria (Hübner, 1788)*: Brăneşti, Pasărea, Pustnicu, 21.V.-8.VIII.19962010, very common.

Rhodostrophia vibicaria (Clerck, 1759): Pasărea, 21.VII.-8.VIII.1996-2009, common. Further records: Bucureşti, Chitila, Comana, Ţigăneşti (Popescu-Gorj, 1964).

Rhodometra sacraria (Linnaeus, 1758): Giurgiu (Popescu-Gorj, 1960).

Lythria purpuraria (Linnaeus, 1758): Brăneşti, Pasărea, 19.VII.-8.VIII.1996-2009, very common. Further records: Mihai Bravu (Popescu, 1939); Bucureşti, Comana, Chitila, Filaret, Jilava (Popescu-Gorj, 1964).

Cataclysme riguata (Hübner, 1813): Pasărea, 30.V.1998, very rare. Further records: Comana, 1888-1891, coll. Salay (Popescu-Gorj, 1964).

Scotopteryx bipunctaria ([Denis \& Schiffermüller], 1775): Comana, 1915-1916 (Popescu-Gorj, 1964).

Scotopteryx mucronata (Scopoli, 1767): Comana, 1915-1916 (Popescu-Gorj, 1964). Cidaria fulvata (Forster,1771)*: Pustnicu, 21.V.2009, common.

Xanthorhoe disignata (Hufnagel, 1767): Bucureşti, Comana, 1913-1916 (PopescuGorj, 1964).

Xanthorhoe fluctuata (Linnaeus, 1758): Brăneşti, Pasărea, Pustnicu, 1-31.V.19962001, common. Further records: Bucureşti, Brăneşti, Comana (Popescu-Gorj, 1964). Epirrhoe alternata (Müller, 1764): Brăneşti, Pasărea, Pustnicu, 21.V.-6.X.1998-2009, common. Further records: Bucureşti, Chitila, Comana, Jilava, Ţigăneşti (PopescuGorj, 1964).

Epirrhoe tristata (Linnaeus, 1758)*: Pustnicu, 21.V.2009, rare.

Epirrhoe rivata (Hübner, 1813): Bucureşti, Chitila (Popescu-Gorj, 1964).

Camptogramma bilineata (Linnaeus, 1758): Pasărea, 30.V.-15.IX.1997-2009, rare. Further records: Bucureşti, Chitila, Comana (Popescu-Gorj, 1964).

Mesoleuca albicillata (Linnaeus, 1758)*: Pasărea, Cernica, Pustnicu, 21-28.VII.19962009, rare.

Pelurga comitata (Linnaeus, 1758): Bucureşti, Comana (Popescu-Gorj, 1964).

Cosmorhoe ocellata (Linnaeus, 1758): Pasărea, 30.V.1998, rare. Further records: Comana, 1915-1916 (Popescu-Gorj, 1964).

Horisme vitalbata ([Denis \& Schiffermüller], 1775)*: Pasărea, 21.VII.1997, very rare.

Horisme tersata ([Denis \& Schiffermüller], 1775)*: Brăneşti, Pasărea, 30-31.V.1998, rare.

Melanthia procellata ([Denis \& Schiffermüller], 1775): Pasărea, 25.VII.1997, very rare.

Epirrita dilutata ([Denis \& Schiffermüller], 1775): Bucureşti (Popescu-Gorj, 1964).

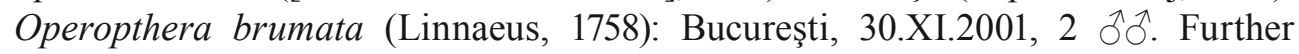
records: Bucureşti, Comana (Popescu-Gorj, 1964). 
Perizoma lugdunaria (Herrich-Schäffer, 1855)*: Pasărea, 21-25.VII.1997, very rare. Perizoma flavofasciata (Thunberg, 1792): Bucureşti, 20.VI.1919 (Popescu-Gorj, 1964).

Eupithecia haworthiata Doubleday, 1856*: Pasărea, Pustnicu 21-30.V.1996-98, common.

Eupithecia linariata ([Denis \& Schiffermüller], 1775): Pasărea, 30.V.-16.IX.19962001, common. Further records: Bucureşti, 6.VI.1888 (Popescu-Gorj, 1964).

Eupithecia laqueraria Herrich-Schäffer, 1848*: Pasărea, 8.VIII.1997, 2 $ぇ$.

Eupithecia centaureata ([Denis \& Schiffermüller], 1775): Brăneşti, Pasărea, 25.VII.9.VIII.1996-2010, common. Further records: Bucureşti (Popescu-Gorj, 1964).

Eupithecia absinthiata (Clerck, 1759)*: Pasărea, 1.VIII.1997, 1 \%.

Eupithecia assimilata Doubleday, 1856: București, 5.IX.1936 (Popescu-Gorj, 1964). Eupithecia subnotata (Hübner, 1803): Bucureşti (Popescu-Gorj, 1964).

Eupithecia millefoliata Rössler, 1866*: Pasărea, Pustnicu, 28.VII.-8.VIII.1996-99, rare.

Eupithecia innotata (Hufnagel,1767)*: Pustnicu, 21.V.2009, rare.

Chloroclystis chloerata (Mabille, 1870)*: Brăneşti, Pasărea, 1-8.VIII.1996-97, rare.

Chloroclystis v-ata (Haworth, 1809)*: Pasărea, 31.VII.-8.VIII.1996-2009, rare.

Aplocera plagiata (Linnaeus, 1758): Pasărea, 15.IX.2001, rare. Further records: București, Chitila (Montandon, 1900; Popescu-Gorj, 1964).

Aplocera praeformata (Hübner, 1826): Mihai Bravu (Popescu, 1939).

Schistostege decussata ([Denis \& Schiffermüller], 1775): Cernica (Popescu-Gorj, 1960); Comana, leg. Montandon (Popescu-Gorj, 1964).

Lithostege griseata ([Denis \& Schiffermüller], 1775): Brăneşti, Pasărea, 21.VII.-8. VIII.1996-2009, common. Further records: Bucureşti (Popescu-Gorj, 1964).

Lithostege farinata (Hufnagel, 1767)*: Pasărea, 30-31.V.1998, rare.

Asthena albulata (Hufnagel, 1767)*: Pasărea, 30.V.-25.VII.1997-2009, rare.

Minoa murinata (Scopoli, 1763): Brăneşti, Comana, Ţigăneşti (Popescu-Gorj, 1964). Lobophora halterata (Hufnagel, 1767)*: Pasărea, 3-4.V.2002, rare.

Family Thaumetopoeidae

Thaumetopoea processionea (Linnaeus, 1758): Bucureşti, 1906-1916, coll. Salay (Popescu-Gorj, 1964).

Family Notodontidae

Clostera curtula (Linnaeus, 1758)*: Pasărea, Cernica, 21.VII.-1.VIII.1996-2001, rare. Clostera pygra (Hufnagel, 1766)*: Pustnicu, 28.VII.2008, 1 ○े. New record for the Romanian Plain.

Cerura vinula (Linnaeus, 1758): Bucureşti, 1892-1819, coll. Salay (Popescu-Gorj, 1964).

Furcula furcula (Clerck, 1759): Bucureşti, Jilava (Popescu-Gorj, 1964).

Dicranura ulmi ([Denis \& Schiffermüller], 1775): Bucureşti, 9.V.1904, coll. Salay (Popescu-Gorj, 1964). 
Ochrostigma velitaris (Hufnagel, 1767): Băneasa, 9.VII.1936 (Nemeş \& Voicu, 1973).

Tritophia tritophus ([Denis \& Schiffermüller], 1775)*: Pasărea, 21.VII.1997, 1 q, Pustnicu, 28.VII.2008, rare.

Notodonta ziczac (Linnaeus, 1758): Pasărea, 3-4.VIII.2002, rare. Further records: Bucureşti (Popescu-Gorj, 1964).

Drymonia dodonaea ([Denis\&Schiffermüller], 1775): Pasărea, Pustnicu, 2130.V.1997-2003, rare. Further records: Bucureşti (Popescu-Gorj, 1964).

Pterostoma palpina (Clerck, 1759): Pustnicu, 28.VII.2008, common. Further records: Bucureşti (Popescu-Gorj, 1964).

Ptilophora plumigera ([Denis \& Schiffermüller], 1775): Bucureşti, 30.XI.2002, 1 §. Further records: Bucureşti (Popescu-Gorj, 1964).

Phalera bucephala (Linnaeus, 1758)*: Pasărea, Pustnicu, 21.V.-8.VIII.1996-2009, common. New record for the Romanian Plain.

Phalera bucephaloides (Ochsenheimer, 1810)*: Comana, Pasărea, Pustnicu, 12-28. VII.1996-2008, common. New record for the Romanian Plain.

Peridea anceps (Goeze, 1781): Bucureşti, 1896-1906, coll. Salay (Popescu-Gorj, 1964).

Stauropus fagi (Linnaeus, 1758)*: Pasărea, Pustnicu, 21.V.-8.VIII.1996-2010, common.

Harpyia milhauseri (Fabricius, 1775): Brăneşti, Pasărea, 21-28.VII.1996-98, rare. Further records: Bucureşti, 9.V.1904, coll. Salay (Popescu-Gorj, 1964).

Spatalia argentina ([Denis \& Schiffermüller], 1775): Brăneşti, Comana, Pasărea, Pustnicu, 7.V.-28.VII.1996-2009, common. Further records: Bucureşti (PopescuGorj, 1964).

\section{Family Noctuidae}

Moma alpium (Osbeck, 1778): Pasărea, Pustnicu, 21.V.-28.VII.1996-2009, rare. Further records: Afumaţi, Bucureşti, 1885-1888 (Popescu-Gorj, 1964; Rákosy, 1996).

Acronicta alni (Linnaeus, 1767): Bucureşti, 1910, coll. Salay (Popescu-Gorj, 1964). Acronicta tridens ([Denis \& Schiffermüller], 1775): Mihai Bravu (Popescu, 1939); Bucureşti, 1892-1912 (Popescu-Gorj, 1964).

Acronicta psi (Linnaeus, 1758): Bucureşti, 1903-1919 (Popescu-Gorj, 1964; Rákosy, 1996).

Acronicta aceris (Linnaeus, 1758): Bucureşti (Popescu-Gorj, 1964; Rákosy, 1996). Acronicta megacephala ([Denis \& Schiffermüller], 1775): Bucureşti, 1916-1928 (Popescu-Gorj, 1964; Rákosy, 1996).

Acronicta auricoma ([Denis \& Schiffermüller], 1775): Bucureşti, 23.VII.1888, leg. F. Salay, (Popescu-Gorj, 1964; Rákosy, 1996).

Acronicta rumicis (Linnaeus, 1758): Pustnicu, 21.V.2009, common. Further records: Bucureşti, Chitila, Comana (Popescu-Gorj, 1964; Rákosy, 1996). 
Craniophora ligustri ([Denis \& Schiffermüller], 1775): Brăneşti, Comana, Pasărea, Pustnicu, 21.V.-9.VIII.1996-2010, very common. Further records: Bucureşti, Chitila, Comana (Popescu-Gorj, 1964; Rákosy, 1996).

Symira nervosa ([Denis \& Schiffermüller], 1775): Bucureşti (Popescu-Gorj, 1964; Rákosy, 1996).

Symira albovenosa (Goeze, 1781): Pasărea, 25.VII.-7.VIII.1996-2001, rare. Further records: Bucureşti, 9.V.1903, coll. Salay (Popescu-Gorj, 1964; Rákosy, 1996).

Cryphia fraudatricula (Hübner, 1803): Pasărea, 25-31.VII.1997-2009, rare, Pustnicu, 28.VII.2008, common. Further records: Bucureşti (Popescu-Gorj, 1964; Rákosy, 1996).

Cryphia algae (Fabricius, 1775): Pasărea, 19.VII.-2.VIII.,1996-2009 common. Further records: Bucureşti (Popescu-Gorj, 1964; Rákosy, 1996).

Cryphia raptricula ([Denis \& Schiffermüller], 1775): Bucureşti (Popescu-Gorj, 1964; Rákosy, 1996).

Idia calvaria ([Denis \& Schiffermüller], 1775): Bucureşti, Comana, Filaret (PopescuGorj, 1964; Rákosy, 1996).

Simplicia rectalis (Eversmann, 1842): Bucureşti, Brăneşti (Popescu-Gorj, 1964; Rákosy, 1996).

Paracolax tristalis (Fabricius, 1794): Brăneşti, Comana, Cernica, Pasărea, 12.VII.-9. VIII. 1996-2009, very common. Further records: Bucureşti (Popescu-Gorj, 1964; Rákosy, 1996).

Herminia grisealis ([Denis \& Schiffermüller], 1775): Bucureşti, Buftea, Comana (Popescu-Gorj, 1964).

Herminia tarsicrinalis (Knoch, 1782): Bucureşti (Rákosy, 1996).

Polypogon tentacularia (Linnaeus, 1758): Bucureşti (Rákosy, 1996).

Zanclognatha lunalis (Scopoli, 1763)*: Pasărea, 21.VII.1997, rare.

Zanclognatha zelleralis (Wocke, 1850)*: Comana, 12.VII.1997, 2 우. New record for the Romanian Plain.

Zanclognatha tarsipennalis Treitschke, 1835*: Brăneşti, Pasărea, 1-8.VIII.1996-98, common.

Pechipogo strigilata (Linnaeus, 1758): Bucureşti, (Rákosy, 1996).

Catocala sponsa (Linnaeus, 1758): Comana, Pasărea, 12-27.VII.1996-2001, rare. Further records: Bucureşti, Comana (Popescu-Gorj, 1964; Rákosy, 1996).

Catocala dilecta (Hübner, 1808) (Fig. 2 C): Comana, 12.VII.1997, 1 ð (Székely \& al., 1998). Further records: Bucureşti (Rákosy, 1996).

Catocala fraxini (Linnaeus, 1758): Afumaţi, 5.VIII.1890, coll. Salay (Popescu-Gorj, 1964).

Catocala nupta (Linnaeus, 1758): Pasărea, Pustnicu, 24.VII.-8.VIII.1996-2010, common. Further records: Bucureşti, Comana (Popescu-Gorj, 1964; Rákosy, 1996). Catocala elocata (Esper, 1787): Brăneşti, Pasărea, Cernica, 19.VII.-8.VIII.19962009, rare. Further records: Mihai Bravu (Popescu, 1939); Bucureşti, Comana (Popescu-Gorj, 1964; Rákosy, 1996). 
Catocala puerpera (Giorna, 1791): Giurgiu (Popescu-Gorj, 1964).

Catocala promissa ([Denis \& Schiffermüller], 1775): Comana, Pustnicu, 12-28. VII.1997-2008, common. Further records: Bucureşti (Popescu-Gorj, 1964; Rákosy, 1996).

Catocala electa (Vieweg, 1783): Bucureşti, Comana, 1893-1899, coll. Salay (PopescuGorj, 1964; Rákosy, 1996).

Catocala conversa (Esper, 1783): Comana (Popescu-Gorj, 1964; Rákosy, 1996). Acceptable historical record, the species being recorded from the northern territories of Bulgaria (Razgrad) (S. Beshkov, pers. comm.).

Catocala nymphagoga (Esper, 1787)* (Fig. 2 G): Comana, Mihai Bravu, Cernica, Pasărea, 12-31.VII.1996-99, very common. New record for the Romanian Plain.

Catocala hymenaea ([Denis \& Schiffermüller], 1775): Pasărea, Cernica, Pustnicu, 19-28.VII.1996-2001, rare. Further records: Pantelimon (Popescu-Gorj, 1964; Rákosy, 1996).

Catocala fulminea (Scopoli, 1763): Bucureşti, Comana, Pantelimon (Popescu-Gorj, 1964; Rákosy, 1996).

Minucia lunaris ([Denis \& Schiffermüller], 1775): Pasărea, Pustnicu, 7-31.V.19972009, rare. Further records: Bucureşti, Comana, Otopeni, Pantelimon (Montandon, 1900; Popescu-Gorj, 1964; Rákosy, 1996).

Dysgonia algira (Linnaeus, 1758): Pasărea, Pustnicu, 25.VII.-9.VIII.1997-2010, rare. Further records: Bucureşti, Pantelimon (Popescu-Gorj, 1964; Rákosy, 1996).

Prodotis stolida (Fabricius, 1775): Brăneşti, Pasărea, Pustnicu, 25.VII.-17.VIII.19962009, rare. Further records: Pantelimon (Popescu-Gorj, 1964; Rákosy, 1996); Mihai Bravu (Popescu, 1939).

Lygephila pastinum (Treitschke, 1820): Bucureşti, Pantelimon, 1901-1904, coll. Salay (Popescu-Gorj, 1964; Rákosy, 1996).

Lygephila craccae ([Denis \& Schiffermüller], 1775): Pasărea, 3-4.VIII.2002, rare. Further records: Bucureşti, Pantelimon, (Popescu-Gorj, 1964; Rákosy, 1996).

Lygephila viciae (Hübner, 1822): Bucureşti (Rákosy, 1996).

Catephia alchymista (Linnaeus, 1758): Pasărea, Pustnicu, 21.V.-1.VIII.1997-2009, very rare. Further records: Bucureşti, 20.VII.1905, coll. Salay, Pantelimon, (PopescuGorj, 1964; Rákosy, 1996).

Aedia funesta (Esper, 1786): Bucureşti, Buftea, Comana, Pantelimon (PopescuGorj, 1964; Rákosy, 1996).

Aedia leucomelas (Linnaeus, 1758): Brăneşti, Pasărea, Pustnicu, 15.V.-6.VIII.19972010, common. Further records: Băneasa, Bucureşti, Giurgiu, Pantelimon (PopescuGorj, 1964; Rákosy, 1996). Subtropical migratory species, distributed in S.-Europe, N.-Africa, Asia Minor, Middle-East, S.-Asia, China, Japan. In Romania it was considered as a rare species, but it has become more and more frequently recorded in the southern part of the country. It is currently known from Muntenia, Dobrogea and the Danube Delta. 
Tyta luctuosa ([Denis \& Schiffermüller], 1775): Brăneşti, Comana, Pasărea, Pustnicu, 21.V.-9.VIII.1996-2010, common. Further records: Bucureşti, Comana, Filaret, Jilava, Pantelimon (Popescu-Gorj, 1964; Rákosy, 1996).

Callistege mi (Clerck, 1759): Pasărea, 3.V.2002, rare. Further records: Comana (Popescu-Gorj, 1964; Rákosy, 1996).

Euclidia glyphica (Linnaeus, 1758): Brăneşti, Pasărea, 25.VII.-1.VIII.1997-2009, rare. Further records: Brăneşti, Comana, Chitila (Popescu-Gorj, 1964; Rákosy, 1996).

Gonospileia triquetra ([Denis \& Schiffermüller], 1775): Ţigăneşti, 13.VIII.1915 (Popescu-Gorj, 1964; Rákosy, 1996).

Laspeyria flexula ([Denis \& Schiffermüller], 1775): Bucureşti (Popescu-Gorj, 1964; Rákosy, 1996).

Scoliopteryx libatrix (Linnaeus, 1758): Pasărea, 8.VIII.1997, 1 ㅇ. Further records: Bucureşti, Comana (Popescu-Gorj, 1964; Rákosy, 1996).

Calyptra thalictri (Borkhausen, 1790): Afumaţi, IX.1880, coll. Salay (Popescu-Gorj, 1964).

Hypena proboscidalis (Linnaeus, 1758): Pasărea, 3.VIII.2002, 1 +. Further records: Bucureşti (Rákosy, 1996).

Hypena rostralis (Linnaeus, 1758): Bucureşti (Popescu-Gorj, 1964; Rákosy, 1996).

Phytometra viridaria (Clerck, 759): Pasărea, 25.VII.-8.VIII.1996-98, rare. Further records: Comana (Popescu-Gorj, 1964; Rákosy, 1996).

Rivula sericealis (Scopoli, 1763): Brăneşti, Pasărea, 30.V.-25.VII.1996-2003, common. Further records: Bucureşti, Comana (Popescu-Gorj, 1964; Rákosy, 1996). Parascotia fuliginaria (Linnaeus, 1761): Pasărea, 25.VII.1997, 1 +. Further records: Bucureşti, 2.VIII.1919 (Popescu-Gorj, 1964; Rákosy, 1996).

Epizeuxis calvaria ([Denis \& Schiffermüller], 1775): Giurgiu (Popescu-Gorj, 1960). Colobochyla salicalis ([Denis \& Schiffermüller], 1775): Pasărea, 25.VII.-1.VIII.19962001, rare. Further records: Comana (Popescu-Gorj, 1964)

Eutelia adulatrix (Hübner, 1813): Bucureşti, 12.IX.1912, coll. Salay (Popescu-Gorj, 1964; Rákosy, 1996).

Lamprotes c-aureum (Knoch, 1781)*: Pasărea, Pustnicu, 25.VII.-1.VIII.1996-99 very rare. New record for the Romanian Plain.

Diachrysia chrysitis (Linnaeus, 1758): Brăneşti, Pasărea, Pustnicu, 21.V.-8.VIII.19962009, rare. Further records: Bucureşti (Popescu-Gorj, 1964, 1977; Rákosy, 1996).

Diachrysia nadeja (Oberthür, 1880): Pasărea, 25.VII.2001, 1 §. Further records: Bucureşti (Rákosy, 1996).

Diachrysia chryson (Esper, 1789): Bucureşti, 1.VIII.1890, coll. Salay (Popescu-Gorj, 1964; Rákosy, 1996).

Macdunnoughia confusa (Stephens, 1850): Pasărea, Pustnicu, 7.V.-8.VIII.19962009, common. Further records: Bucureşti, Chitila, Comana (Popescu-Gorj, 1964; Rákosy, 1996).

Plusia festucae (Linnaeus, 1758): Brăneşti, Pasărea, 17.VII.-2.VIII.1996-99, common. Further records: Bucureşti (Popescu-Gorj, 1964; Rákosy, 1996). 
Autographa gamma (Linnaeus, 1758): Brăneşti, Pasărea, Pustnicu, 21.V.3.X.1996-2010, common. Further records: Bucureşti, Comana, Jilava (PopescuGorj, 1964; Rákosy, 1996).

Autographa bractea ([Denis \& Schiffermüller], 1775): Chitila, 8.IX.1910, coll. Salay (Popescu-Gorj, 1964; Rákosy, 1996).

Plusidia cheiranthi (Tauscher, 1809) (Fig. 2 K): Cislău, 21.VI.-9.VII.1997-99, common. Further records: Bucureşti, 1880-1935 (Popescu-Gorj, 1964; Rákosy, 1996).

Trichoplusia ni (Hübner, 1803): Brăneşti, Pasărea, 6-20.X.1997-99, very rare. Further records: Bucureşti (Rákosy, 1996).

Chrysodeixis chalcites (Esper, [1789])* (Fig. 2 J): Pasărea, 1.VIII.2000, 1 q, (Székely \& Stanciu, 2002).

Subtropical migratory species widely distributed across Eurasia, Africa, Oceania and Australia. In Europe it is resident only in the southern parts. Recorded for the first time in Romania two decades ago (Rákosy \& Neumann, 1990), the species seems to have become more and more common during the last years. Although it is currently known in Romania only from Dobrogea and Muntenia (Rákosy \& Neumann, 1990; Székely \& Stanciu, 2002; Dincă, 2006; Székely, Dincă \& Juhász, 2011). New record for the Romanian Plain.

Abrostola asclepiadis ([Denis \& Schiffermüller], 1775)*: Brăneşti, Comana, Pasărea, Pustnicu, 21.V.-8.VIII.1996-2010, very common.

Abrostola triplasia (Linnaeus, 1758) (=trigemina Werneburg, 1864): Brăneşti, Pasărea, 30.V.-8.VII.1997-2009, common. Further records: Bucureşti (Montandon, 1900; Popescu-Gorj, 1964).

Emmelia trabealis (Scopoli, 1763): Brăneşti, Comana, Pasărea, Pustnicu, 21.V.-9. VIII.1996-2010, very common. Further records: Mihai Bravu (Popescu, 1939); Bucureşti, Comana, Jilava (Popescu-Gorj, 1964).

Tarachidia candefacta (Hübner, [1831])* (Fig. 2 E, L): Manolache (surroundings of Bucharest, Ilfov county), 11.V.2010 (1 spec., leg. Cristian Mihai). It is a species of Nearctic origin described from Pennsylvania (USA). It is widely distributed in North America, ranging from southern Canada to Mexico. Tarachidia candefacta has been intentionally introduced in southern Russia (the Krasnodar region) in the years 60 of the 20th century as a biological control measure against the invasive Ambrosia artemisiifolia (Schurov, 1998; Poltavsky \& Artokhin, 2006; Poltavsky et al., 2008). The species is therefore rapidly expanding to the west through the steppes north of the Black Sea (Hacker, Legrain \& Fibiger, 2008). Recently reported that new for the fauna of Romania (Székely, Dincă \& Juhász, 2011). The specimen from Manolache represent the first records of T. candefacta from Muntenia and confirm its expansion to the west. T. candefacta has already been collected in Bulgaria on the southern banks of the Danube next to the Romanian border (appreciatively south of the Romanian town Corabia) (S. Beshkov, pers. comm.). New record for the Romanian Plain. 
Acontia lucida (Hufnagel, 1776): Brăneşti, Pasărea, 25.VII.-8.VIII., rare. Other remarks: Bucureşti, Comana (Montandon, 1900; Popescu-Gorj, 1964; Rákosy, 1996).

Phylophilla obliterata (Rambur, 1833)*: Pasărea, Cernica, 30.V.-1.VIII.1996-99, common.

Protodeltote pygarga (Hufnagel, 1766)*: Pasărea, Pustnicu, 21.V.-2.VIII.1997-2009, common.

Deltote uncula (Clerck, 1759): Pasărea, 26.V.2001, rare. Further records: Bucureşti (Popescu-Gorj, 1964; Rákosy, 1996).

Deltote bankiana (Fabricius, 1775): Pasărea, 17-25.VII.1996-99, very rare. Further records: Călugăreni (Popescu-Gorj, 1964; Rákosy, 1996).

Pseudeustrotia candidula ([Denis \& Schiffermüller], 1775)*: Pasărea, 25.VII.1997, rare. Further records: Bucureşti (Rákosy, 1996).

Calymma communimacula ([Denis \& Schiffermüller], 1775): Pasărea, Cernica, 25.VII.-11.VIII.1997-99, rare. Further records: București (Rákosy, 1996).

Eublemma respersa (Fabricius, 1794): Pasărea, 24.VII.-7.VIII.1997-99, rare. Further records: București (Popescu-Gorj, 1964; Rákosy, 1996).

Eublemma purpurina ([Denis \& Schiffermüller], 1775)*: Pasărea, Cernica, Pustnicu, 24.VII.-8.VIII.1996-2009, common. New record for the Romanian Plain.

Trisateles emortualis ([Denis \& Schiffermüller], 1775)*: Pasărea, 25-31.VII.1996-98, rare. New record for the Romanian Plain.

Cucullia absinthii (Linnaeus, 1758)*: Pasărea, 8.VIII.1997, 1 9. New record for the Romanian Plain.

Cucullia umbratica (Linnaeus, 1758): Pasărea, Cernica, 24-31.VII.1997-99, rare. Further records: Bucureşti (Popescu-Gorj, 1964; Rákosy, 1996).

Cucullia tanaceti ([Denis \& Schiffermüller], 1775): Bucureşti, 18.V.1904, coll. Salay (Popescu-Gorj, 1964; Rákosy, 1996).

Shargacucullia lychnitis (Rambur, 1833): Chitila, 20.V.1884, coll. Salay (PopescuGorj, 1964; Rákosy, 1996).

Shargacucullia blattariae (Esper, 1790): Bucureşti (Rákosy, 1996).

Shargacucullia verbasci (Linnaeus, 1758): Pasărea, 3.V.1999, 1 ठ․ Further records: Bucureşti, 20.IV.1911 (Popescu-Gorj, 1964; Rákosy, 1996).

Shargacucullia scrophulariae ([Denis \& Schiffermüller], 1775)*: Pustnicu, 21.V.2009, 2 ô. New record for the Romanian Plain.

Shargacucullia prenanthis Boisduval, 1850: Bucureşti (Rákosy, 1996).

Calophasia lunula (Hufnagel, 1766): Pasărea, Cernica, 24-25.VII.1996-99, rare. Further records: Bucureşti, Comana (Popescu-Gorj, 1964; Rákosy, 1996).

Pyrois cinnamomea (Goeze, 1781): Afumaţi, 30.VIII.1880, leg. Siebrecht (Salay, 1910; Rákosy, 1996).

Amphipyra pyramidea (Linnaeus, 1758): Pasărea, Pustnicu, 28.VII.-8.VIII.19962008, rare. Further records: Bucureşti (Popescu-Gorj, 1964; Rákosy, 1996). 
Amphipyra berbera svenssoni Fletscher, 1968: Afumaţi, Bucureşti, Comana (Popescu-Gorj, 1977).

Amphipyra livida ([Denis \& Schiffermüller], 1775)*: Pasărea, Pustnicu, 28.VII.-2. VIII.1997, rare.

Amphipyra tragopoginis (Clerck, 1759): Pasărea, 1-8.VIII.1997-99, rare. Further records: Bucureşti (Rákosy, 1996).

Asteroscopus sphinx (Hufnagel, 1766): Bucureşti, 15.X.1916 (Popescu-Gorj, 1964; Rákosy, 1996).

Diloba caeruleocephala (Linnaeus, 1758): Pasărea, Bucureşti, 20.X.-24.XI.2001, rare. Further records: Bucureşti (Popescu-Gorj, 1964; Rákosy, 1996).

Panemeria tenebrata (Scopoli, 1763): Bucureşti (Popescu-Gorj, 1964).

Aegle koekeritziana (Hübner, 1759): Pasărea, 26.V.2001., very rare. Further records: Bucureşti (Popescu-Gorj, 1964; Rákosy, 1996).

Schinia scutosa ([Denis \& Schiffermüller], 1775): Brăneşti, Cernica, Pasărea, Pustnicu, 21.VII.-9.VIII.1996-2009, common. Further records: Bucureşti, Ţigăneşti (Popescu-Gorj, 1964).

Heliothis viriplaca (Hufnagel, 1766): Bucureşti, Brăneşti, Comana, Jilava (PopescuGorj, 1964; Rákosy, 1996).

Heliothis maritima bulgarica Draudt, 1938: Pasărea, 1.VIII.2004, 1 đ. Further records: Bucureşti, Comana, (Popescu-Gorj, 1964; Rákosy, 1996).

Heliothis peltigera ([Denis \& Schiffermüller], 1775): Pasărea, 1-8.VIII.1996-2001, rare. Further records: Mihai Bravu (Popescu, 1939; Rákosy, 1996).

Helicoverpa armigera (Hübner, 1808)*: Pasărea, Pustnicu, 21.VII.-8.VIII.19962008, common.

Pyrrhia umbra (Hufnagel, 1766): Pasărea, Pustnicu, Cernica, 30.V.-21.VII.1996-99, rare. Further records: Bucureşti (Rákosy, 1996).

Periphanes delphinii (Linnaeus, 1758) (Fig. 2 I): Pasărea, Cernica, Pustnicu, 21.VII.9.VIII.1997-99, rare. Further records: Bucureşti (Popescu-Gorj, 1964; Rákosy, 1996). Elaphria venustula (Hübner, 1790)*: Brăneşti, Pasărea, Pustnicu, 28.VII.-9. VIII.1996-2009, rare. New record for the Romanian Plain.

Caradrina morpheus (Hufnagel, 1766)*: Pasărea, Cernica, 24-31.VII.1996-99, rare. Platyperigea kadenii (Freyer, 1836): Pasărea, 3.VIII.2002, 2 ふึે. Further records: Bucureşti (Popescu-Gorj, 1964; Rákosy, 1996).

Paradrina clavipalpis (Scopoli, 1763): Bucureşti, Chitila, Comana (Popescu-Gorj, 1964; Rákosy, 1996).

Hoplodrina blanda ([Denis \& Schiffermüller], 1775)*: Pasărea, 15.IX.2001, rare. New record for the Romanian Plain.

Hoplodrina octogenaria (Goeze, 1781): Bucureşti (Rákosy, 1996).

Hoplodrina ambigua ([Denis \& Schiffermüller], 1775)*: Pasărea, Pustnicu, 2130.V.1996-99, rare.

Hoplodrina respersa ([Denis \& Schiffermüller], 1775): Bucureşti (Rákosy, 1996). 
Charanyca trigammica (Hufnagel, 1766): Brăneşti, Pasărea, 21.VII.-8.VIII.19962010, very common. Further records: Bucureşti, Comana (Popescu-Gorj, 1964).

Spodoptera exigua (Hübner, 1808): Pasărea, 8.VIII.1997, 1 ô. Further records: Bucureşti (Rákosy, 1996).

Chilodes maritima (Tauscher, 1806)*: Pasărea, 26.V.2001, 1 ô. New record for the Romanian Plain.

Athetis gluteosa (Treitschke, 1835)*: Pasărea, Pustnicu, 28.VII.-8.VIII.1996-2001, rare. Further records: Comana (Rákosy, 1996).

Dypterygia scabriuscula (Hufnagel, 1766): Pasărea, Pustnicu, 28.VII.-8.VIII.19972009, common. Further records: Bucureşti, 12.VI.1928 (Popescu-Gorj, 1964; Rákosy, 1996).

Thalpophila matura (Hufnagel, 1766)*: Pasărea, 15-16.IX.2001, rare.

Trachea atriplicis (Linnaeus, 1758): Pasărea, 30.V.-2.IX.1996-2010, rare. Further records: Bucureşti, Comana (Popescu-Gorj, 1964; Rákosy, 1996).

Euplexia lucipara (Linnaeus, 1758): Pasărea, 26.V.-17.VIII.1997-2009, rare. Further records: Afumaţi, Bucureşti, Comana (Popescu-Gorj, 1964; Rákosy, 1996).

Phlogophora meticulosa (Linnaeus, 1758): Pasărea, 15-16.IX.2001, rare. Further records: Bucureşti, Chitila (Popescu-Gorj, 1964; Rákosy, 1996).

Actinotia polyodon (Clerck, 1759): Pasărea, Cernica, 30.V.-31.VII.1997-2009, rare. Further records: Bucureşti (Rákosy, 1996).

Enargia abluta (Hübner, 1808)**: Bucureşti, 1911-1928, coll. Salay (Popescu-Gorj, 1964).

Ipimorpha subtusa ([Denis \& Schiffermüller], 1775): Bucureşti, 1911-1928, coll. Salay (Popescu-Gorj, 1964).

Mesogona acetosellae ([Denis \& Schiffermüller], 1775): Pasărea, 20.X.2001, rare. Further records: Bucureşti, 1915-1919 (Popescu-Gorj, 1964).

Mesogona oxalina (Hübner, 1803): Bucureşti (Rákosy, 1996).

Dycicla oo (Linnaeus, 1758): Bucureşti (Rákosy, 1996).

Parastichtys suspecta (Hübner, 1817): Bucureşti (Rákosy, 1996).

Cosmia diffinis (Linnaeus, 1767)*: Comana, Pasărea, 12.VII.-1.VIII., rare. New record for the Romanian Plain.

Cosmia pyralina ([Denis \& Schiffermüller], 1775): Pasărea, 19.VII.-15.IX.1996-99, very common. Further records: Bucureşti, 1889, coll. Salay (Popescu-Gorj, 1964).

Cosmia affinis (Linnaeus, 1758): Comana (Rákosy, 1996).

Cosmia trapezina (Linnaeus, 1758): Pasărea, Cernica, Pustnicu, 30.V.-9.VIII.19972009, common. Further records: Bucureşti, Chitila, Comana (Popescu-Gorj, 1964; Rákosy, 1996).

Atethmia centrago (Haworth, 1803)*: Pasărea, 15-16.IX.2001, common. New record for the Romanian Plain.

Atethmia ambusta ([Denis \& Schiffermüller], 1775): Pasărea, 15-16.IX.2001, rare. Further records: Bucureşti (Rákosy, 1996). 
Xanthia togata (Esper, 1788)*: Pasărea, 6.X.2001, 1 đิ. Further records: Comana (Rákosy, 1996).

Xanthia sulphurago ([Denis \& Schiffermüller], 1775)*: Pasărea, 15.IX.-6.X.2001, very common. New record for the Romanian Plain.

Xanthia gilvago ([Denis \& Schiffermüller], 1775)*: Pasărea, 15.IX.-6.X.2001, common. Further records: Comana (Rákosy, 1996).

Xanthia citrago (Linnaeus, 1758): Pasărea, 6.X.2001, rare. Further records: Comana (Rákosy, 1996).

Agrochola lychnidis ([Denis \& Schiffermüller], 1775): Pasărea, 15.IX.-20.X.2001, rare. Further records: Bucureşti, Chitila (Popescu-Gorj, 1964; Rákosy, 1996).

Agrochola circellaris (Hufnagel, 1766): Pasărea, 15.IX.-6.X.2001, common. Further records: Bucureşti, 1891-1906, coll. Salay (Popescu-Gorj, 1964; Rákosy, 1996). Agrochola lota (Clerck, 1759)*: Pasărea, 15.IX.-6.X.2001, common. New record for the Romanian Plain.

Agrochola macilenta (Hübner, 1809)*: Pasărea, 6.X.2001, common. New record for the Romanian Plain.

Agrochola nitida ([Denis \& Schiffermüller], 1775): Pasărea, 6-20.X.2001, rare. Further records: Bucureşti (Rákosy, 1996).

Agrochola helvola (Linnaeus, 1758): Pasărea, 15.IX.-6.X.2001, rare. Further records: Comana (Rákosy, 1996).

Agrochola litura (Linnaeus, 1758): Pasărea, 6-20.X.2001, common. Further records: Comana (Rákosy, 1996).

Agrochola laevis (Hübner, 1803)*: Pasărea, 6.X.2001, rare.

Eupsilia transversa (Hufnagel, 1766): Bucureşti, Pasărea, 15.IX.-6.X.2001, rare. Further records: Comana (Popescu-Gorj, 1964; Rákosy, 1996).

Jodia craceago ([Denis \& Schiffermüller], 1775): Bucureşti, 1890-1916 (Montandon, 1900; Popescu-Gorj, 1964; Rákosy, 1996).

Conistra vaccinii (Linnaeus, 1761): Pasărea, 15.IX.-20.X.2001, common. Further records: Bucureşti, Chitila (Popescu-Gorj, 1964; Rákosy, 1996).

Conistra rubiginea ([Denis \& Schiffermüller], 1775): Bucureşti, 1889-1896, coll. Salay (Popescu-Gorj, 1964; Rákosy, 1996).

Conistra ligula (Esper, 1791): Bucureşti, 1.VII.1900, coll. Salay (Popescu-Gorj, 1964).

Orbona fragariae (Vieweg, 1790)**: Bucureşti, 1885-1889, coll. Salay (PopescuGorj, 1964).

Ulochlaena hirta (Hübner, 1813)**: Bucureşti (Montandon, 1900), Bucureşti, 6.XI.1929 (Popescu-Gorj, 1964). Acceptable historical record, the species being recorded from the northern territories of Bulgaria (Danube River, Somovit near Gulyantsi) (S. Beshkov, pers. comm.).

Aporophyla lutulenta ([Denis \& Schiffermüller], 1775): Pasărea, 6-20.X.2001, rare. Further records: Comana (Rákosy, 1996). 
Lithopane ornithopus (Hufnagel, 1766): Pasărea, 3-4.V.2002, rare. Further records: Bucureşti, Comana (Popescu-Gorj, 1964; Rákosy, 1996).

Xylena exsoleta (Linnaeus, 1758)*: Pasărea, 3.V.2002,1 中. New record for the Romanian Plain.

Allophyes oxyacanthae (Linnaeus, 1758): Pasărea, 20.X.2001, rare. Further records: Comana (Rákosy, 1996).

Dichonia aprilina (Linnaeus, 1758): Bucureşti, VII.1882, coll. Salay (Popescu-Gorj, 1964).

Ammoconia caecimacula ([Denis \& Schiffermüller], 1775): Pasărea, 6-20.X.2001, common. Further records: Bucureşti, 1911-1932 (Popescu-Gorj, 1964; Rákosy, 1996). Valeria oleagina ([Denis \& Schiffermüller], 1775): Bucureşti (Rákosy, 1996). Apamea crenata (Hufnagel, 1766)*: Pasărea, 30-31.V.1998, rare. New record for the Romanian Plain.

Apamea remissa (Hübner, 1809): Bucureşti (Rákosy, 1996).

Eremobina pabulatricula (Brahm, 1791)*: Comana, 12.VII.1997, 4 ふぇં, 2 우. New record for the Romanian Plain.

Oligia strigilis (Linnaeus, 1758): Bucureşti (Popescu-Gorj, 1964, Rákosy, 1996).

Oligia latruncula ([Denis \& Schiffermüller], 1775): Pasărea, 30.V.1998, common. Further records: Bucureşti, Comana (Popescu-Gorj, 1964; Rákosy, 1996).

Oligia versicolor (Borkhausen, 1775)*: Comana, Pasărea, 30.V.-12.VII.1996-99, common.

Mesoligia furuncula ([Denis \& Schiffermüller], 1775): Bucureşti, 1915-1919 (Popescu-Gorj, 1964; Rákosy, 1996).

Mesapamea secalis (Linnaeus, 1758)*: Pasărea, 25-31.VII.1997-99, rare. New record for the Romanian Plain.

Luperina testacea ([Denis \& Schiffermüller], 1775)*: Pasărea, 15.IX.2001, 1 §. New record for the Romanian Plain.

Rhizedra lutosa (Hübner, 1803): Bucureşti, coll. Salay (Popescu-Gorj, 1964).

Amphipoea oculea (Linnaeus, 1758): Pasărea, 1.VIII.1997, 1 ¡. Further records: Comana, 1915-1916 (Popescu-Gorj, 1964).

Gortyna flavago ([Denis \& Schiffermüller], 1775)*: Pasărea, 1.VIII.-15.IX.19982001, rare. New record for the Romanian Plain.

Calamia tridens (Hufnagel, 1766)*: Pasărea, 1.VIII.2001, 1 đ.

Staurophora celsia (Linnaeus, 1758)**: Bucureşti (Rákosy, 1996).

Nonagria typhae (Thunberg, 1784): Pasărea, 25.VII.2001, 1 q. Further records: Bucureşti, 21.X.1905, coll. Salay (Popescu-Gorj, 1964).

Discestra trifolii (Hufnagel, 1766): Brăneşti, Pasărea, 25.VII.-15.IX.1996-2010, common. Further records: Bucureşti (Popescu-Gorj, 1964).

Lacanobia w-latinum (Hufnagel, 1766): Brăneşti, Pasărea, 1-9.VIII.1996-2002, rare. Further records: Bucureşti, Comana (Popescu-Gorj, 1964).

Lacanobia splendens (Hübner, 1808)*: Pasărea, 30.V.1997, 1 đ. 
Lacanobia oleracea (Linnaeus, 1758): Brăneşti, Pasărea, Pustnicu, 28.VII.-9. VIII.1996-2009, rare. Further records: Bucureşti, Chitila, Comana, Filaret, Jilava, Țigăneşti (Popescu-Gorj, 1964).

Lacanobia thalassina (Hufnagel, 1766): București, Comana (Popescu-Gorj, 1964).

Lacanobia suasa ([Denis \& Schiffermüller], 1775): Bucureşti, Comana (PopescuGorj, 1964).

Lacanobia blenna (Hübner, 1824): Bucureşti, 25.VIII.1932 (König, 1975).

Hecatera dysodea ([Denis \& Schiffermüller], 1775): Pasărea, 25-31.VII.1997-2008, rare. Further records: București (Popescu-Gorj, 1964).

Hecatera bicolorata (Hufnagel, 1766)*: Pasărea, 1.VIII.1997, 1 ô, Pustnicu, 28.VII.2008, rare. New record for the Romanian Plain.

Hecatera cappa (Hübner, [1803])*: Pasărea, 21-22.VII.1997, rare. New record for the Romanian Plain. Distributed in S.-Europe, Asia Minor, S.-Ukraine, S.-Russia, Altai. In Romania known only from Oltenia, Muntenia and Dobrogea.

Hadena bicruris (Hufnagel, 1766)*: Pasărea, 1.VIII.1997, 1 đ.

Hadena luteago ([Denis \& Schiffermüller], 1775): Pasărea, 3-4.VIII.2002, rare. Further records: Bucureşti (Popescu-Gorj, 1964).

Hadena albimacula (Borkhausen, 1792)*: Pasărea, 21.VII.1997, 2 $\delta^{\lambda}$. New record for the Romanian Plain.

Hadena rivularis (Fabricius, 1775): București (Popescu-Gorj, 1964).

Hadena perplexa ([Denis \& Schiffermüller], 1775): Bucureşti (Popescu-Gorj, 1964). Melanchra persicariae (Linnaeus, 1758): Comana (Popescu-Gorj, 1964; Rákosy, 1996).

Mamestra brassicae (Linnaeus, 1758): Pasărea, Pustnicu, 24.VII.-8.VIII.1996-2009, rare. Further records: Bucureşti, Chitila, Comana, Filaret, Ţigăneşti (Popescu-Gorj, 1964).

Mythimna turca (Linnaeus, 1758): Pasărea, Cernica, 30.V.-8.VIII.1997-2008, common. Further records: Bucureşti (Rákosy, 1996).

Mythimna ferrago (Fabricius, 1787): Brăneşti, Pasărea, 21.VII.-16.VIII.1996-2008, rare. Further records: Comana (Popescu-Gorj, 1964; Rákosy, 1996).

Mythimna albipuncta ([Denis \& Schiffermüller], 1775): Brăneşti, Pasărea, Pustnicu, 21.V.-20.X.1996-2010, common. Further records: Bucureşti, Chitila, Comana (Popescu-Gorj, 1964).

Mythimna vitellina (Hübner, 1790): Brăneşti, Pasărea, Cernica, Pustnicu, 21.V.20.X.1996-2010, common. Further records: Bucureşti (Rákosy, 1996).

Mythimna impura (Hübner, 1808): Afumaţi, 1880, coll. Salay (Popescu-Gorj, 1964). Mythimna pallens (Linnaeus, 1758): Brăneşti, Comana, Pasărea, 12.VII.-8.VIII.19962010, rare. Further records: Bucureşti (Rákosy, 1996).

Mythimna obsoleta (Hübner, 1803): Pasărea, 25-30.V.1996-99, rare. Further records: Comana (Rákosy, 1996).

Mythimna comma (Linnaeus, 1758)*: Pasărea, 25.VII.-1.VIII.1997-99, rare. New record for the Romanian Plain. 
Mythimna l-album (Linnaeus, 1758): Pasărea, 1.VIII.-6.X.1996-2009, rare. Further records: Bucureşti, Chitila, Comana (Popescu-Gorj, 1964).

Orthosia incerta (Hufnagel, 1766): Pasărea, 3-26.V.1997-2010, rare. Further records: Comana (Rákosy, 1996).

Orthosia gothica (Linnaeus, 1758): Pasărea, Pustnicu, 3-21.V,1997-2010 common. Further records: Bucureşti, Comana (Popescu-Gorj, 1964; Rákosy, 1996).

Orthosia cruda ([Denis \& Schiffermüller], 1775): Pasărea, 2-3.V.2001, rare. Further records: Bucureşti (Popescu-Gorj, 1964; Rákosy, 1996).

Orthosia miniosa ([Denis \& Schiffermüller], 1775): Brăneşti, 2.IV.1903, coll. Salay (Popescu-Gorj, 1964, Rákosy, 1996).

Orthosia cerasi (Fabricius, 1775): Pasărea, Pustnicu, 3-21.V.1997-2009, common. Further records: Bucureşti (Popescu-Gorj, 1964; Rákosy, 1996).

Egira conspicillaris (Linnaeus, 1758): Pasărea, Pustnicu, 3-21.V.1997-2009, common. Further records: Bucureşti (Popescu-Gorj, 1964; Rákosy, 1996).

Tholera decimalis (Poda, 1761): Pasărea, 15.IX.2001, rare. Further records: Bucureşti, Comana (Popescu-Gorj, 1964).

Axylia putris (Linnaeus, 1758): Pasărea, Pustnicu, 30.V.-1.VIII.1996-2009, rare. Further records: Bucureşti, Chitila (Popescu-Gorj, 1964).

Ochropleura plecta (Linnaeus, 1758): Brăneşti, Pasărea, 21.V.-15.IX.1996-2010, common. Further records: Bucureşti, Chitila (Popescu-Gorj, 1964).

Diarsia brunnea ([Denis \& Schiffermüller], 1775)*: Pasărea, 19.VII.2001, 1 §.

Diarsia dahlii (Hübner, 1813): Bucureşti (Rákosy, 1996).

Noctua pronuba (Linnaeus, 1758): Brăneşti, Comana, Pasărea, Pustnicu, 21.V.20.X.1996-2009, common. Further records: Bucureşti, Chitila, Comana (PopescuGorj, 1964; Rákosy, 1996).

Noctua orbona (Hufnagel, 1766): Pasărea, 31.VII.-16.VIII.1996-2009, rare. Further records: Bucureşti (Rákosy, 1996).

Noctua fimbriata (Schreber, 1759): Comana, Pasărea, Pustnicu, 12.VII.-7.VIII.19962009, rare. Further records: Bucureşti, Comana (Rákosy, 1996).

Noctua janthina ([Denis \& Schiffermüller], 1775): Brăneşti, Pasărea, 21.VII.-15. IX.1996-99, rare. Further records: Bucureşti (Popescu-Gorj, 1964; Rákosy, 1996).

Noctua haywardi (Tams, 1926)* (Fig. 2 H): Pasărea, 19.VII.2001 and 1.VIII.2001, 2 うへ。.

Signaled as a new species for the Romanian fauna in 2002 (Székely \& Stanciu, 2002). A West-Asian element, spread on the Balkan Peninsula (in Greece, Bulgaria, Croatia, Macedonia), the south of Hungary, the Crimean Peninsula (Fibiger, 1993; Efetov \& Budashkin, 1990), Cyprus and the western territories of Turkey, a very rare species, known in the Romanian fauna only from this area. New record for the Romanian Plain.

Spaelotis ravida ([Denis \& Schiffermüller], 1775): Bucureşti, 12.VII.1919 (PopescuGorj, 1960, 1964; Rákosy, 1996). 
Rhyacia simulans (Hufnagel, 1766): Brăneşti, Pasărea, 2.VIII.-15.IX.1997-2001, rare. Further records: Bucureşti, 2.VII.1960 (König, 1975; Rákosy, 1996).

Opigena polygona ([Denis \& Schiffermüller], 1775): Bucureşti, 5.VI.1889, coll. Salay (Popescu-Gorj, 1964).

Eugraphe sigma ([Denis \& Schiffermüller], 1775): Bucureşti (Rákosy, 1996).

Xestia c-nigrum (Linnaeus, 1758): Pasărea, Cernica, Pustnicu, 21.V.-15.IX.19962009, common. Further records: Bucureşti, Comana (Popescu-Gorj, 1964).

Xestia ditrapezium ([Denis \& Schiffermüller], 1775): Pasărea, 21.VII.-1.VIII.19972009, rare. Further records: Bucureşti (Rákosy, 1996).

Xestia triangulum (Hufnagel, 1766): Pasărea, 19.VII.-1.VIII.1997-2009, rare. Further records: Bucureşti (Rákosy, 1996).

Xestia rhomboidea (Esper, 1790)*: Pasărea, 7.VIII.1997, 1 q.

Xestia xanthographa ([Denis \& Schiffermüller],1775)*: Pasărea, 15.IX.-6.X. 2001, very common. New record for the Romanian Plain.

Cerastis rubricosa ([Denis \& Schiffermüller], 1775): Pustnicu, 21.V.2009, common. Further records: Bucureşti, 13.VI.1916 (Popescu-Gorj, 1964; Rákosy, 1996).

Cerastis leucographa ([Denis \& Schiffermüller], 1775)*: Pustnicu, 21.V.2009, common. New record for the Romanian Plain.

Naenia typica (Linnaeus, 1758): Afumaţi, Bucureşti, 1891-1906, coll. Salay (Popescu-Gorj, 1964).

Anaplectoides prasina ([Denis \& Schiffermüller], 1775): Comana, 1890-1916 (Popescu-Gorj, 1964; Rákosy, 1996).

Peridroma saucia (Hübner, 1808): Brăneşti, Pasărea, 15.VIII.-2.X.1997-2002, rare. Further records: Bucureşti (Popescu-Gorj, 1964; Rákosy, 1996).

Actebia praecox (Linnaeus, 1758): Bucureşti, 1913, coll. Salay (Popescu-Gorj, 1964; Rákosy, 1996).

Euxoa aquilina ([Denis \& Schiffermüller], 1775): Comana, 12.VII.1997, 1 ð. Further records: Bucureşti, 7.VII.1928 (Popescu-Gorj, 1964; Rákosy, 1996).

Euxoa obelisca ([Denis \& Schiffermüller], 1775): Bucureşti (Rákosy, 1996).

Euxoa temera (Hübner, 1808): Pasărea, 15.IX.2001, 1 §. Further records: Băneasa (Popescu-Gorj, 1960).

Euxoa nigricans (Linnaeus, 1758): Pasărea, 27.V.2001, 1 §. Further records: Comana, 30.VI.1894 (Popescu-Gorj, 1964; Rákosy, 1996).

Euxoa tritici (Linnaeus, 1758): Comana, 12.VII.1997, rare. Further records: Bucureşti (Rákosy, 1996).

Agrotis crassa (Hübner, 1803): Pasărea, 1.VIII.-15.IX.1997-2001, common. Further records: Bucureşti (Rákosy, 1996).

Agrotis ipsilon (Hufnagel, 1766): Pasărea, Pustnicu, 3.V.-15.IX., common. Further records: Bucureşti, Chitila, Comana (Popescu-Gorj, 1964).

Agrotis exclamationis (Linnaeus, 1758): Brăneşti, Pasărea, Cernica, Pustnicu, 21.V.9.VIII.1996-2010, common. Other remarks: Mihai Bravu (Popescu, 1939); Bucureşti, Chitila, Comana (Popescu-Gorj, 1964). 
Agrotis segetum ([Denis \& Schiffermüller], 1775): Brăneşti, Cernica, Pustnicu, 1.V.2.X.1996-2010, common. Further records: Mihai Bravu (Popescu, 1939); Bucureşti, Chitila, Comana (Popescu-Gorj, 1964).

Calocasia coryli (Linnaeus, 1758): Pasărea, Pustnicu, 3-26.V.1997-2009, rare. Further records: Comana, 1915-1924 (Popescu-Gorj, 1964; Rákosy, 1996).

Family Lymantriidae

Lymantria monacha (Linnaeus, 1758)**: Comana, 16.VII.1916 (Popescu-Gorj, 1964). Lymantria dispar (Linnaeus, 1758): Brănești, Comana, Pasărea, Pustnicu, 12.VII.17.VIII.1996-2010, common. Further records: Brăneşti, Comana, Bucureşti, Chitila, Jilava, Ţigăneşti (Popescu-Gorj, 1964).

Callitaera pudibunda (Linnaeus, 1758)*: Pustnicu, 21.V.2009, common. New record for the Romanian Plain.

Dicallomera fascelina (Linnaeus, 1758)**: Bucureşti, 1906-1919, coll. Salay (Popescu-Gorj, 1964).

Orgyia antiqua (Linnaeus, 1758): Brăneşti, Pasărea, Pustnicu, 21.VII.-9.VIII.19972008, common. Further records: Bucureşti, Comana (Popescu-Gorj, 1964).

Euproctis chrysorrhoea (Hübner, 1822): Pasărea, 25.VII.-9.VIII.1996-2009, rare. Further records: Bucureşti, Brăneşti, Comana, Chitila, Ţigăneşti (Popescu-Gorj, 1964).

Euproctis similis (Fuessly, 1767): Pasărea, Cernica, 21.VII.-11.VIII.1996-2010, rare. Further records: Bucureşti, Brăneşti, Chitila, Comana, Ţigăneşti (Popescu-Gorj, 1964).

Penthophera morio (Linnaeus, 1758): Afumaţi, 8.V.1881, coll. Salay (Popescu-Gorj, 1964).

Leucoma salicis (Linnaeus, 1758): Comana, Pasărea, 12.VII.-1.VIII.1996-99 common. Further records: Bucureşti, Chitila, Comana, Ţigăneşti (Popescu-Gorj, 1964).

Arctornis l-nigrum (Müller, 1764)*: Comana, Pasărea, Cernica, Pustnicu, 12.VII.-8. VIII.1996-2010, very common.

Family Nolidae

Meganola togatulalis (Hübner, 1798): Bucureşti (Popescu-Gorj, 1964; Rákosy, 1996).

Meganola strigula ([Denis \& Schiffermüller], 1775)*: Brăneşti, Pustnicu, Pasărea, Cernica, 21.V.-9.VIII.1998-2009, very common. New record for the Romanian Plain. Meganola kolbi (Daniel,1935)*: Pasărea, 30.V.1998, 2 ऊิ 0 . New record for the Romanian Plain.

Nola cuculatella (Linnaeus, 1758)*: Pasărea, 8.VIII.1997, 1 q. New record for the Romanian Plain.

Nola cristatula (Hübner, 1793)*: Brăneşti, Pasărea, 7-8.VIII.1997, rare. New record for the Romanian Plain. 
Nycteola asiatica (Krulikowsky, 1904)*: Pasărea, 19-26.VII.2001, rare. Further records: Comana (Rákosy, 1996).

Bena bicolorana (Fuessly, 1775) (=prasinana auct., nec Linnaeus): Brăneşti, Pasărea, 25-31.VII.1997-2009, rare. Further records: Bucureşti (Rákosy, 1996).

Pseudoips prasinanus (Linnaeus, 1758) (=faganus Fabricius, 1781): Brăneşti, Pasărea, 31.V.-25.VII.1997-2008, rare. Further records: Bucureşti (Popescu-Gorj, 1964; Rákosy, 1996).

Earias clorana (Linnaeus, 1758): Pasărea, 25-31.VII.1997-99, rare. Further records: Bucureşti (Popescu-Gorj, 1964; König, 1975; Rákosy, 1996).

Earias vernana (Fabricius, 1787)*: Pasărea, 21.VII.-8.VIII.1997-99, rare. New record for the Romanian Plain.

Family Arctiidae

Miltochrista miniata (Forster, 1771) (Fig. 2 U): Brăneşti, Comana, Pustnicu, Cernica, 21.V.-16.VIII.1996-2010. very common. Further records: Băneasa (PopescuGorj, 1964); Miltochrista miniata - form. fasciata, Rebel, 1915 (Fig. 2 U): Pasărea, Brăneşti, Pustnicu, rare.

Cybosia mesomella (Linnaeus, 1758): Brăneşti, Pasărea, 26-27.V.2001, very common. Further records: Chitila, Ţigăneşti (Popescu-Gorj, 1964).

Pelosia muscerda (Hufnagel, 1766)*: Pasărea, Pustnicu, 26.V.-1.VIII.1997-2001, common.

Pelosia obtusa (Herrich-Schäffer, 1847)*: Pasărea, 17.VII.-1.VIII.1996-2002, rare. New record for the Romanian Plain.

Atolmis rubicollis (Linnaeus, 1758): Pasărea, 21.VII.-8.VIII.1996-2001, rare. Further records: Bucureşti (Popescu-Gorj, 1964).

Lithosia quadra (Linnaeus, 1758): Brăneşti, Pasărea, 21.VII.-15.IX.1996-2008, rare. Further records: Brăneşti, Comana, Ţigăneşti (Popescu-Gorj, 1964).

Eilema complana balcanica (Daniel, 1939)*: Pasărea, 25.VII.-8.VIII.1996-99, rare. New record for the Romanian Plain.

Eilema pseudocomplana (Daniel, 1939)*: Pasărea, 7-8.VIII.1997, 2 ふో . New record for the Romanian Plain.

Eilema sororcula (Hufnagel, 1766): Pasărea, Pustnicu, 25.VII.-8.VIII.1997-2009, common. Further records: Comana, 1914-1915, coll. Salay (Popescu-Gorj, 1964).

Amata phegea (Linnaeus, 1758): Otopeni, Româneşti, Săftica, 15.VI.2009, very common. Further records: Bucureşti, Chitila, Filaret, Jilava (Montandon, 1900; Popescu-Gorj, 1964).

Dysauxes ancilla (Linnaeus, 1767): Pasărea, 25-31.VII.1996-99, rare. Further records: Bucureşti, Comana (Popescu-Gorj, 1964)

Phragmatobia fuliginosa (Linnaeus, 1758): Pasărea, Pustnicu, 21.V.-8.VIII.19962010, rare. Further records: Bucureşti, Comana (Popescu-Gorj, 1964)

Spilosoma lutea (Hufnagel, 1766): Brăneşti, Pasărea, Cernica, 21.VII.-9.VIII.199699, common. Further records: Bucureşti, Comana (Popescu-Gorj, 1964). 
Spilosoma lubricipeda (Linnaeus, 1758): Brănești, Comana, Pasărea, Pustnicu, 12.VII.-9.VIII.1996-2009, very common. Further records: Comana (Popescu-Gorj, 1964).

Spilosoma urticae (Esper, 1789): Bucureşti, Comana, Filaret (Popescu-Gorj, 1964). Hyphantria cunea (Drury, 1773)*: Pasărea, Pustnicu, 21.VII.-6.VIII.1997-2001, rare. Diaphora mendica (Clerck, 1759): Bucureşti, Chitila (Popescu-Gorj, 1964).

Rhyparia purpurata (Linnaeus, 1758): Comana, 26.VI.1918 (Popescu-Gorj, 1964).

Diacrisia sannio (Linnaeus, 1758): Pasărea, 3-4.VIII.2002, common. Further records: Comana, Chitila, Țigăneşti (Popescu-Gorj, 1964).

Arctia caja (Linnaeus, 1758)*: Pasărea, 3.VIII.2004, 1 §. New record for the Romanian Plain.

Arctia villica (Linnaeus, 1758): Pasărea, 26-27.V.2001, rare. Further records: Bucureşti, Chitila (Popescu-Gorj, 1964).

Callimorpha dominula (Linnaeus, 1758)**: Comana, 1911-1916 (Popescu-Gorj, 1964).

Euplagia quadripunctaria (Poda, 1761) (Fig. 2 T): Pasărea, 25.VII.-7.VIII.1996-2001, rare. Further records: Bucureşti, Mihai Bravu (Popescu, 1939; Popescu-Gorj, 1964).

Family Eriocraniidae

Eriocrania subpurpurella (Haworth, 1828): Băneasa, Pasărea, Ştefăneşti, leg. A. Popescu-Gorj (Kovács \& Kovács, 1998).

\section{Family Hepialidae}

Triodia sylvina (Linnaeus, 1758): Brăneşti, Pasărea, 15-16.IX.2001, very common. Further records: Bucureşti, Ţigăneşti (Popescu-Gorj, 1964).

Family Nepticulidae

Ectoedemia lousiella (Sircom,1849): Bucuresti (Drăghia, 1970).

Family Incurvariidae

Incurvaria pectinea (Haworth, 1828): Pasărea, 29.VIII.1972, leg. I. Drăghia (Kovács \& Kovács, 2000).

Incurvaria masculella ([Denis \& Schiffermüller], 1775): Păd. Andronache, Mogoşoaia, Săbăreni (Kovács \& Kovács, 2000).

\section{Family Tineidae}

Euplecamus anthracinalis (Scopoli, 1763): Pasărea, 21.VII.-8.VIII.1997-99, rare. Further records: Bucureşti, Păd. Andronache, Comana, Pantelimon (Căpuşe, 1968). Morophaga boleti (Fabricius, 1777): Bucureşti (Căpuşe, 1968).

Neurothaumasia ankerella (Mann, 1867): Pasărea, 8.VIII.1997, 1 §.. Further records: Bucureşti (Căpuşe, 1968).

Nemaxera emortuella (Zeller, 1839): Băneasa (Căpuşe, 1968). 
Nemapogon granellus (Linnaeus, 1758): Bucureşti (Căpuşe, 1968).

Nemapogon cloacellus (Haworth, 1828): București (Montandon, 1900; Căpuşe, 1968).

Nemapogon personellus (Pierce \& Metcalfe, 1934): Bucureşti (Căpuşe, 1968).

Haplotinea ditella (Pierce \& Metcalfe, 1934): Bucureşti (Căpuşe, 1968).

Cilicornella relicinella (Herrich-Schäffer, 1851): Bucureşti, Comana (Căpuşe, 1968).

Tricophaga tapetzella (Linnaeus, 1758): Bucureşti (Căpuşe, 1968).

Tineola biseliella (Hummel, 1828): Bucureşti (Căpuşe, 1968).

Niditinea fuscipunctella (Haworth, 1828): Bucureşti (Căpuşe, 1968).

Tinea pelionella Linnaeus, 1758: Bucureşti (Căpuşe, 1968).

Tinea leonhardi Petersen, 1957: Bucureşti (Căpuşe, 1968).

Tinea turicensis Müller-Rutz, 1920: Bucureşti (Căpuşe, 1968).

Tinea lanella Pierce \& Metcalfe, 1934: Bucureşti (Căpuşe, 1968).

Monopis rusticella (Hübner, 1776): Bucureşti (Căpuşe, 1968).

Monopis monachella (Hübner, 1776): Pasărea, 8.VIII.1997, 1 ex. Further records: Bucureşti, Ştefăneşti (Căpuşe, 1968).

Monopis crocicapitella (Clemens, 1859): Bucureşti, Ştefăneşti (Căpuşe, 1968).

Monopis imella (Hübner, 1813): Bucureşti (Căpuşe, 1968).

Tenaga rhenania (G. Petersen, 1962): Bucureşti (Căpuşe, 1968).

Monopis nigricantella (Milliere, 1872): București (Caradja, 1901; Căpuşe, 1968).

Infurcitinea ignicomella (Herrich-Schäffer, 1851): Bucureşti (Montandon, 1900; Căpuşe, 1968).

Infurcitinea albicomella (Herrich-Schäffer, 1851): Comana, Mironeşti (Căpuşe, 1968).

\section{Family Psychidae}

Psychidea nudella (Ochsenheimer, 1810): Bucureşti (Caradja, 1903).

Epichnopterix plumella ([Denis \& Schiffermüller], 1775): Bucureşti (Popescu-Gorj, 1964).

Caneophora hirsuta (Poda, 1761): Pasărea, 6.VI.1998, ex. larva, 1 §. (leg. S. M. Stanciu). Further records: Afumaţi, Bucureşti (Popescu-Gorj, 1964).

Pachytelia vilosella (Ochsenheimer, 1810): Bucureşti, Filaret (Popescu-Gorj, 1964). Megalophanes viciella ([Denis \& Schiffermüller], 1775)*: Pasărea, 30.V.1998, 1 ○ૈ. Sterrhoptrix fusca (Haworth, 1809)*: Pasărea, 30.V.-25.VII.1996-99, common.

\section{Family Gracillaridae}

Callisto denticulella (Thunberg, 1794)*: Brăneşti, Pasărea, 25.VII.-7.VIII.1996-99, rare.

Phyllonorycter platani (Staudinger, 1870): Bucureşti (Drăghia, 1970; Rákosy \& Momeu, 2009).

Cameraria ochridella Deschka \& Dimic, 1986: Bucureşti (Rákosy, Goia \& Kovács, 2003). 
Family Yponomeutidae

Yponomeuta evonymella (Linnaeus, 1758)*: Brăneşti, Pasărea, 21.VII.-8.VIII.199699, common.

Yponomeuta padella (Linnaeus, 1758): Bucureşti, Chitila, Comana (Popescu-Gorj, 1964), Pasărea, 13.VI.1960 (König, 1975).

Yponomeuta malinellus Zeller, 1838*: Brăneşti, Pasărea, 31.VII.-8.VIII.1996-99, common.

Yponomeuta irrorella (Hübner, 1796): Bucureşti, Comana (Popescu-Gorj, 1964).

Yponomeuta plumbella ([Denis \& Schiffermüller], 1775): Pasărea, 8.VIII.1997, 1 †. Further records: Bucureşti, Comana (Popescu-Gorj, 1964).

Family Ypsolophidae

Ypsolophus scabrella (Linnaeus, 1758)*: Pasărea; 1.VIII.1998, 1 §ิ.

Family Plutellidae

Plutella xylostella (Linnaeus, 1758): Brăneşti, Pasărea, 25-31.VII.1996-2001, common. Further records: Pasărea, 7.V.1946, 17.VII.1960 (König, 1975).

Eidophasia messingiella (Fischer v. Röslerstamm, 1840): Pasărea, 30.V.1998, common. Further records: Pasărea, 9.VI.1961 (König, 1975).

Family Ethmiidae

Ethmia funerella (Fabricius, 1787)*: Pasărea, 30.V.1998, common.

Ethmia bipunctella (Fabricius, 1775)*: Pasărea, 30.V.1998, rare.

Family Depressariidae

Agonopteryx propinquella (Treitschke, 1835): Bucureşti (Popescu-Gorj, 1964).

Agonopteryx alstromeriana (Clerck, 1759): Pasărea, 1.VIII.1998, 1 đ.. Further records: Băneasa, 29.VIII.1960 (König, 1975).

Family Chimabachidae

Diurnea fagella ([Denis \& Schiffermüller], 1775): Păd. Andronache, 18-23.III.1936 (König, 1975).

Family Oecophoridae

Epicallima formosella ([Denis \& Schiffermüller], 1775): Brăneşti, Pasărea, 30 31.V.1997-99, very common. Other remarks: Bucureşti (Popescu-Gorj, 1964).

Esperia oliviella (Fabricius, 1794): Băneasa, 12.VI.1950, 18.VII.1961 (König, 1975). Alabonia staintoniella (Zeller, 1850): Băneasa, 24.V.1961 (König, 1975).

Harpella forficella (Scopoli, 1763)*: Brăneşti, Pasărea, 21.VII.-8.VIII.1997-99, rare. Carcina quercana (Fabricius, 1775)*: Pasărea, 7.VIII.1997, 1 o. 
Family Lecithoceridae

Lecithocera nigrana (Duponchel, 1836): Bucureşti (Popescu-Gorj, 1964).

Homalexastis briantella (Turati, 1879)*: Pasărea, 21-25.VII.1996-99, rare.

Family Coleophoridae

Coleophora frischella (Linnaeus, 1758)*: Pasărea, Pustnicu, 31.V.-9.VIII.1996-2001, rare.

Coleophora mayrella (Hübner, 1813): Bucureşti (Montandon, 1900).

Family Pterolonchidae

Pterolonche albescens Zeller, 1847: Pasărea, 1.VIII.2001, 2 $\widehat{\partial}$. Further records: Pasărea, 18.VII.1978 (Popescu-Gorj, 1986).

Family Argyresthiidae

Argyresthia arcella (Fabricius, 1771)*: Pasărea, 31.VII.-7.VIII.1997-2001, very common.

Family Autostichidae

Oegoconia quadripuncta (Haworth, 1828): Bucureşti, 5.VI.1947 (König, 1975).

Family Amphisbatidae

Hypercallia citrinalis (Scopoli, 1763)*: Pasărea, 30.V.1998, 1 đ..

Anchinia daphnella ([Denis \& Schiffermüller], 1775)*: Pasărea, 1.VIII.2001, 1 đે.

Family Cosmopterigidae

Pyroderces argyrogrammos (Zeller, 1847): Bucureşti (Popescu-Gorj, 1964).

Family Gelechiidae

Pseudotelphusa scalella (Scopoli, 1763): Băneasa, 13.VIII.1961 (König, 1975).

Helcistogramma trianulella (Herrich-Schäffer, 1854): Bucureşti (Popescu-Gorj, 1964).

Atremaea lonchoptera (Staudinger, 1871)*: Comana, 12.VII.1997, rare.

\section{Family Limacodidae}

Apoda limacodes (Hufnagel, 1766)*: Pasărea, Pustnicu, 21.V.-28.VII.1996-2009, common.

Family Zygaenidae

Rhagades pruni ([Denis \& Schiffermüller], 1775): Bucureşti, Comana (PopescuGorj, 1964).

Jordanita globulariae (Hübner, 1793)*: Pasărea, 25.VII.-6.VIII.1996-99, rare. 
Zygaena purpuralis pluto Ochsenheimer, 1808: Comana, Ţigăneşti (Popescu-Gorj, 1964).

Zygaena loti balcanica Reiss, 1922: Bucureşti, Comana (Popescu-Gorj, 1964).

Zygaena carniolica caliacrae Reiss, 1931: Bucureşti, Chitila, Comana (PopescuGorj, 1964).

Zygaena viciae dacica Caradja, 1893: Comana (Popescu-Gorj, 1964).

Zygaena filipendulae (Linnaeus, 1758): Brăneşti, Comana, Ţigăneşti (Popescu-Gorj, 1964).

Zygaena lonicerae (Scheven, 1777): Comana (Popescu-Gorj, 1964).

Zygaena angelicae transcarpathina Hormuzachi, 1902: Comana (Popescu-Gorj, 1964).

Zygaena contaminei Boisduval, 1834: Comana (Popescu-Gorj, 1964).

Zygaena ephialtes pannonica Holik, 1972: Bucureşti, Comana, Filaret (PopescuGorj, 1964).

Family Sesiidae

Sesia apiformis (Clerck, 1759): Bucureşti (Popescu-Gorj, 1964).

Paranthrene tabaniformis (Rottenburg, 1775): Bucureşti, Chitila (Popescu-Gorj, 1964).

Synanthedon tipuliformis (Clerck, 1759): Filaret-Bucureşti (Popescu-Gorj, 1964).

Synanthedon vespiformis (Linnaeus, 1758): Căldăruşani, Pasărea (Popescu-Gorj, 1964).

Synanthedon conopiformis (Esper, 1782)*: Pustnicu, 21.V. 2009, 2 §ోळे.

Bembecia scopigera (Scopoli, 1763): Comana (Popescu-Gorj, 1964).

Chamaesphecia annellata (Zeller, 1847): București, Comana (Popescu-Gorj, 1964). Chamaesphecia masariformis (Ochsenheimer, 1808): Comana (Popescu-Gorj, 1964).

Chamaesphecia empiformis (Esper, 1783): Comana, Pasărea (Popescu-Gorj, 1964).

Family Cossidae

Cossus cossus (Linnaeus, 1758): Pasărea, Pustnicu, 21.VII.-6.VIII.1996-2009, rare. Further records: Bucureşti (Popescu-Gorj, 1964).

Zeuzera pyrina (Linnaeus, 1761): Brăneşti, Pasărea, Cernica, Pustnicu, 21.VII.-9. VIII.1996-2010, common. Further records: Bucureşti, Ţigăneşti (Popescu-Gorj, 1964).

Phragmataecia castaneae (Hübner, 1790): Pasărea, Pustnicu, 30.V.-28.VII.19972009, rare. Further records: Bucureşti (Popescu-Gorj, 1964).

Parahypopta caestrum (Hübner, 1808): Pasărea, 2.VIII.2002, 1 §. Further records: Bucureşti (Montandon, 1900). 
Family Tortricidae

Pterochroa schreibersiana (Fröhlich, 1828): Păd. Andronache, 28.IX.1936, leg. A. Popescu-Gorj (Kovács \& Kovács, 2001).

Agapeta hamana (Linnaeus, 1758): Pasărea, 1.VIII.1998, 1 q. Further records: Bucureşti (Popescu-Gorj, 1964).

Agapeta zoegana (Linnaeus, 1758)*: Pasărea, 1-8.VIII.1998, rare.

Cochylidia phaleratana (Herrich-Schäffer, 1840)*: Pasărea, 1-8.VIII.1998, rare.

Eugnosta magnificana (Rebel, 1914)*: Pasărea, 30.V.1998, 1 §. Further records: Pasărea (Popescu-Gorj, 1974).

Eugnosta lathoniana (Hübner, 1800)*: Pasărea, 30.V.1998, rare.

Tortrix viridana (Linnaeus, 1758): Brăneşti, Pasărea, 30-31.V.1996-2010, common. Further records: Bucureşti (Popescu-Gorj, 1964).

Acleris holmiana (Linnaeus, 1758)*: Pasărea, 25-31.VII.1996-99, rare.

Acleris forsskaleana (Linnaeus, 1758): Pasărea, 25-31.VII.1997-99, rare. Further records: Pasărea, 20.VII.1973 (König, 1975).

Acleris lorquiniana (Duponchel, 1835): Pasărea, 5.VIII.1971, 1 q (Karisch, 2004).

Acleris kochiella (Goeze, 1783): Pasărea, 5.VIII.1971, 1 ô (Karisch, 2004).

Argyrothaenia pulchellana (Haworth, 1811)*: Pasărea, 21-31.VII., common.

Cnephasia communnana (Herrich-Schäffer, 1851): Brăneşti, Pasărea, 30-31.V.19972001, rare. Further records: Pasărea, 7.V.1961 (König, 1975).

Sparganothis pilleriana ([Denis \& Schiffermüller], 1775): Bucureşti, 10.VIII.1936 (König, 1975).

Archips podana (Scopoli, 1763): Pasărea, 30.V.1998, common. Further records: Bucureşti, Filaret (Montandon, 1900; Popescu-Gorj, 1964); Pasărea (König, 1975). Archips rosana (Linnaeus, 1758)*: Pasărea, 21-25.VII.1997-99, common. Further records: Pasărea, 16.VI.197(?), 1 đิ (Karisch, 2010).

Archips crataegana (Hübner, 1799)*: Pasărea, 30.V.1998, rare.

Ptycholoma lacheana (Linnaeus, 1758): Păd. Andronache, 28.V.1913 (König, 1975).

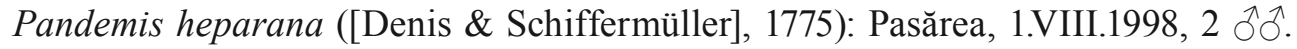
Further records: Băneasa, 21.VIII.1961 (König, 1975).

Bactra lanceolana (Hübner, 1799)*: Pasărea, 1-8.VIII.1997-98, rare.

Bactra furfurana (Haworth, 1811)*: Pasărea, 1-8.VIII.1997-98, rare.

Celypha striana ([Denis \& Schiffermüller], 1775): Brăneşti, Pasărea, 1-8.VIII.,199799 rare. Further records: Bucureşti (Montandon, 1900), Băneasa, 15.VIIII.1959 (König, 1975).

Celypha lacunana ([Denis \& Schiffermüller], 1775): Bucureşti (Popescu-Gorj, 1964).

Celypha rivulana (Scopoli, 1763): Bucureşti (Popescu-Gorj, 1964).

Hedya nubiferana (Haworth, 1811)*: Pasărea, 30.V.1998, rare.

Aleimma loeflingiana (Linnaeus, 1758)*: Pasărea, 30.V.1998, rare.

Olethreutes arcuella (Clerck, 1759): Pasărea, 30.V.1998, 1 ô. Further records: Băneasa, 6.V.1951 (König, 1975). 
Notocelia uddmanniana (Linnaeus, 1758)*: Pasărea, 30-31.V.1998-2001, rare.

Thiodia citrana (Hübner, 1799)*: Brăneşti, Pasărea, 25.VII.-8.VIII.1997-99, rare.

Epinotia festivana (Hübner, 1799): Băneasa, Bucureşti (Popescu-Gorj, 1974).

Eucosma metzneriana (Treitschke, 1830)*: Comana, Pasărea, 12-25.VII.1997-99, rare.

Eucosma pupillana (Clerck, 1759): Pasărea, 27.VI.1954 (König, 1975).

Epiblema scutulana ([Denis \& Schiffermüller], 1775): Pasărea, 25.VII.-8.VIII.199799, common. Further records: Bucureşti (Popescu-Gorj, 1964).

Epiblema foenella (Linnaeus, 1758)*: Pasărea, 25.VII.1997, rare.

Ancylis comptana (Fröhlich, 1828): Băneasa, 18.V.1960 (König, 1975).

Ancylis badiana ([Denis \& Schiffermüller], 1775)*: Pasărea, 30.V.1998, rare.

Ancylis mitterbacheriana ([Denis \& Schiffermüller], 1775)*: Brăneşti, Pasărea, 3031.V.1997-99, rare.

Cydia splendana (Hübner, 1799)*: Pasărea, 1-8.VIII.1997-99, rare.

Cydia pomonella (Linnaeus, 1758): Brăneşti, Pasărea, 1-9.VIII.1996-2009, very common. Further records: Bucureşti (Popescu-Gorj, 1964).

Cydia fagiglandana (Zeller, 1841)*: Pasărea, 1-8.VIII.1997-99, rare.

Lathronympha strigana (Fabricius, 1775)*: Pasărea, 1-8.VIII.1996-2001, rare.

Choristoneura hebenstriatella (Müller, 1764)*: Pasărea, 30.V.1998, rare.

Phalonidia contractana (Zeller, 1847)*: Pasărea, 7-8.VIII.1997, rare. Further records: București, 5.VII.1973 (Karisch, 1999).

Phalonidia manniana (Fischer von Röslerstamm, 1839): Pasărea, 5.VIII.1971 (Karisch, 1999).

Gynnidomorpha permixtana (Denis \& Schiffermüller], 1775): Pasărea, 6.VIII.1973 (Karisch, 2003).

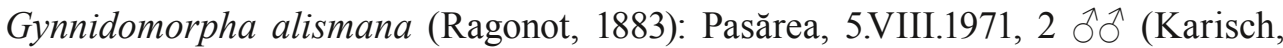
1999).

Aethes francillana (Fabricius, 1794): Bucureşti, 5.VII.1973, 3 ふోग, 1 우 (Karisch, 1999).

Cochylidia implicitana (Wocke, 1856): Pasărea, Bucureşti, 5-6.VII.1972, 2 ex. (Karisch, 1999, 2003).

Cochylis epilinana Duponchel, 1842: Pasărea, 5.VIII.1971, 1 ○ (Karisch, 1999).

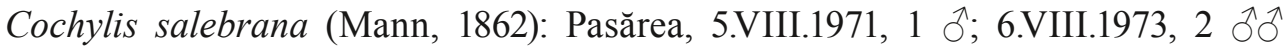
(Karisch, 1999).

Cochylis posterana Zeller, 1847: Bucureşti, 5.VII.1973, 1 đે, Pasărea, 5.VIII.1971, 1 ô (Karisch, 1999).

Family Alucitidae

Alucita hexadactyla (Linnaeus, 1758)*: Pasărea, 6.X.2001, 2. ex. 
Family Pterophoridae

Oxyptilus chrysodactyla ([Denis \& Schiffermüller], 1775): Pasărea, 21.VII.1997, 1 §. Further records: Bucureşti (Montandon, 1900), Chitila (Popescu-Gorj, 1964). Pterophorus tridactyla (Linnaeus, 1758)*: Pasărea, Cernica, 21-24.VII.1996-99, rare. Pterophorus pentadactyla (Linnaeus, 1758): Pasărea, 30.V.-1.VIII.1997-99, common. Further records: Bucureşti (Popescu-Gorj, 1964); Păd. Andronache, Băneasa (König, 1975).

Emmelina monodactyla (Linnaeus, 1758): Bucureşti, Comana (Popescu-Gorj, 1964; König, 1975).

Stenoptilia pterodactyla (Linnaeus, 1761): Comana (Popescu-Gorj, 1964).

Family Pyralidae

Subfamily Galeriinae

Melissoblaptes zelleri Joannis, 1932: Pasărea, 1-8.VIII.1996-2001, rare. Further records: Bucureşti (Popescu-Gorj, 1964).

Lamoria annella ([Denis \& Schiffermüller], 1775)*: Pasărea, 1.VIII.2001, 1 đે.

Galleria mellonella (Linnaeus, 1758): Pasărea, 6.X.2001, 1 §. Further records: Bucureşti (Popescu-Gorj, 1964).

\section{Subfamily Pyralinae}

Synaphe moldavica (Esper, 1794): Păd. Andronache, 6.VI.1937 (König, 1975).

Pyralis farinalis (Linnaeus, 1758): Brăneşti, Comana, Pasărea, 12.VII.-9.VIII.19962001, common. Further records: Bucureşti (Popescu-Gorj, 1964; König, 1975).

Pyralis regalis ([Denis \& Schiffermüller], 1775)*: Brăneşti, Pasărea, 7-9.VIII.199699, very rare.

Aglossa signicostalis Staudinger, 1871: Comana (Popescu-Gorj, 1964).

Aglossa caprealis (Hübner, 1809): Bucureşti, Comana (Popescu-Gorj, 1964; König, 1975).

Aglossa pinguinalis (Linnaeus, 1758): Pasărea, 15.IX.2001, rare. Further records: București, Pasărea (Popescu-Gorj, 1964; König, 1975).

Hypsospygia costalis (Fabricius, 1775): Pasărea, 21.VII.-9.VIII.1996-99, common. Further records: Bucureşti (Popescu-Gorj, 1964).

Herculia rubidalis ([Denis \& Schiffermüller], 1775): Pasărea, 21.VII.1997., rare. Further records: Păd. Andronache, 13.VI.1934 (König, 1975).

Herculia incarnatalis (Zeller, 1847)*: Pasărea, 21.VII.1997, 1 సે.

Orthopygia glaucinalis (Linnaeus, 1758): Pasărea, 21.VIII.-11.VIII.1997-99, rare. Further records: Bucureşti (Popescu-Gorj, 1964); Păd. Andronache (König, 1975). Endotricha flammealis ([Denis \& Schiffermüller], 1775): Brăneşti, Pasărea, 21.VII.9.VIII.1997-99, very common. Further records: Bucureşti, Păd. Andronache, 19401958 (König, 1975). 
Subfamily Phycitinae

Trachonitis cristella ([Denis \& Schiffermüller], 1775): Pasărea, 6.VI.1960 (König, 1975).

Elegia similella (Zinken, 1818): Băneasa, 8.VIII.1962 (König, 1975).

Etiella zinkenella (Treitschke, 1832): Brăneşti, Pasărea, 21.VII.-9.VIII.1996-99, very common. Further records: Păd. Andronache (König, 1975).

Oncocera semirubella (Scopoli, 1763): Brăneşti, Comana, Pasărea, 12.VII.-9. VIII.1996-2010, common. Further records: Bucureşti, Chitila, Filaret, Băneasa, 23.VI.1948, 23.VII.1939 (König, 1975).

Oxybia transversella (Duponchel, 1836)*: Brăneşti, Comana, Pasărea, 12.VII.-9. VIII.1996-99, very common.

Pempelia formosa (Haworth, 1811): Pasărea, 21.VII.1997, 1 §. Further records: Ştefăneşti, 7.VI.1961 (König, 1975).

Psorosa dahliella (Treitschke, 1832): Bucureşti, 15.VI.1947 (König, 1975).

Diorycrtria abietella ([Denis \& Schiffermüller], 1775)*: Brăneşti, Pasărea, 30.V.-8. VIII.1996-2001, very common.

Phycita metzneri (Zeller, 1846): Pasărea (Popescu-Gorj, 1964).

Hypochalcia dignella (Hübner, 1799): Giurgiu (Popescu-Gorj, 1960).

Epischnia prodromella (Hübner, 1799): Giurgiu, 6.IX.1946 (König, 1975).

Nyctegretis achatinella (Hübner, 1824)*: Pasărea, 30.V.1998, rare.

Nephopterix angustella (Hübner, 1769): Băneasa, 14.VI.1946, 2.IX.1949 (König, 1975).

Trachycera advenella (Zincken, 1818): Băneasa, 7.VIII.1960 (König, 1975).

Episcythrastis tetricella ([Denis \& Schiffermüller], 1775): Pasărea, 21.VII.-9. VIII.,1997-99 common. Further records: Păd. Andronache, 21.VI.1947 (König, 1975). Myelois circumvoluta (Fourcroy, 1785): Pasărea, 27.VII.-8.VIII.1997-2008, common. Further records: Pasărea, 12.VI.1948 (König, 1975).

Gymnancyla canella ([Denis \& Schiffermüller], 1775): Bucureşti (Popescu-Gorj, 1964).

Euzophera pinguis (Haworth, 1811)*: Pasărea, 25.VII.1997, 1 đે.

Euzophera bigella (Zeller, 1848): Bucureşti (Popescu-Gorj, 1964).

Homoeosoma sinuella (Fabricius, 1794)*: Brăneşti, Pasărea, 1-8.VIII.1996-2009, rare.

Homoeosoma nebulella ([Denis \& Schiffermüller], 1775): Brăneşti, Pasărea, 31.VII.9.VIII.1996-99, rare. Further records: Bucureşti (Popescu-Gorj, 1964).

Plodia interpunctella (Hübner, 1813): Pasărea, 25.VII.1997, 1 §. Further records: Bucureşti (Popescu-Gorj, 1964; König, 1975).

Ephestia elutella (Hübner, 1796): Bucureşti (Popescu-Gorj, 1964; König, 1975).

Anerastria lotella (Hübner, 1813)*: Pasărea, 21.VII.1997, 1 స.

Ematheudes punctella (Treitschke, 1833): Pasărea, 1-8.VIII.1996-99, rare. Further records: Bucureşti (Popescu-Gorj, 1964). 
Subfamily Scopariinae

Scoparia manifestella (Herrich-Schäffer, 1848): Pasărea, 30.V.1998., rare. Further records: Bucureşti (Popescu-Gorj, 1964).

Scoparia mercurella (Linnaeus, 1758): București (Popescu-Gorj, 1964).

Subfamily Crambinae

Euchromius ocellea (Haworth, 1811)*: Pasărea, 1-8.VIII.1997-2002, rare.

Euchromius bella (Hübner, 1796): Brăneşti, Pasărea, Cernica, 21.VII.-9.VIII.199699, very common. Further records: Băneasa (König, 1975).

Chilo phragmitella (Hübner, 1824)*: Pasărea, 25.VII.1997, 1 ô.

Friedlanderia cicatricella (Hübner, 1824): Comana, 20.VII,1963 (König, 1975).

Agriphila tristella ([Denis \& Schiffermüller], 1775): Pasărea, 1-8.VIII.1997-2002, rare. Further records: Comana (Popescu-Gorj, 1964).

Agriphila inquinatella ([Denis \& Schiffermüller], 1775): Comana (Popescu-Gorj, 1964); Pasărea, 13.VII.1960 (König, 1975).

Catoptria pinella (Linnaeus, 1758)*: Pasărea, 21.VII.-8.VIII.1997-98, rare.

Calamotropha paludella (Hübner, 1824): Bucureşti (Montandon, 1900); Pasărea, 1.VIII.1997, rare.

Crambus ericellus (Hübner, 1813)*: Pasărea, 1-2.VIII.1997, rare.

Chrysocrambus linetellus (Fabricius, 1781)*: Pasărea, 21.VII.-8.VIII.1997-98, common.

Chrysocrambus craterella (Scopoli, 1763): Comana, Pasărea, 12.VII.-8.VIII.199799, common. Further records: Băneasa, 9.VI.1946 (König, 1975).

Pediasia contaminella (Hübner, 1796)*: Pasărea, 1-8.VIII.1997-99, rare.

Subfamily Schoenobiinae

Schoenobius gigantella Duponchel, 1836: Snagov, 24.VII.1957 (König, 1975).

Scirpophaga praelata (Scopoli, 1763): Pasărea, 25.VII.-8.VIII.1996-2009, rare. Further records: Bucureşti, 8.VII.1974 (König, 1975).

Subfamily Acentropinae

Elophila nymphaeta (Linnaeus, 1758): Bucureşti (Montandon, 1900); Bucureşti, Chitila (König, 1975).

Acentria ephemerella ([Denis \& Schiffermüller], 1775): Giurgiu, 1.VII.1946 (König, 1975).

Cataclysta lemnata (Linnaeus, 1758): Pasărea, 25.VII.-8.VIII.1996-99, common. Further records: Bucureşti, Păd. Andronache, 1935-1950 (König, 1975).

Parapoynx stratiotata (Linnaeus, 1758): Pasărea, 25.VII.-8.VIII.1997-99, common. Further records: Bucureşti (Popescu-Gorj, 1964; König, 1975).

Parapoynx nivalis ([Denis \& Schiffermüller], 1775): Bucureşti (Popescu-Gorj, 1964). 
Subfamily Odontinae

Aporodes floralis (Hübner, 1809): Pasărea, 1.VIII.1997, 1 ठ̊. Further records: Pasărea, 22.VII.1958 (König, 1975).

Cynaeda dentalis ([Denis \& Schiffermüller], 1775)*: Pasărea, 7.VIII.1997, 1 ô. Epacestria pustulalis (Hübner, 1823)*: Pasărea, 25.VII.-11.VIII.1997-98, rare.

Titanio normalis (Hübner, 1796): Păd. Andronache, 9-21.VIII.1945 (König, 1975).

Subfamily Evergestinae

Evergestis frumentalis (Linnaeus, 1758)*: Pasărea, 1-8.VIII.1996-99, rare.

Evergestis forficalis (Linnaeus, 1758)*: Pasărea, 1-8.VIII.1997-99, very rare.

Evergestis extimalis (Scopoli, 1763): Pasărea, Cernica, 24-31.VII.1996-2009, rare. Further records: Băneasa, 7.VIII.1960 (König, 1975).

Evergestis limbata (Linnaeus, 1758)*: Pasărea, 30.V.1998, very rare.

\section{Subfamily Pyraustinae}

Udea ferrugalis (Hübner, 1796): Brăneşti, 9.VIII.1997, 1 ठ․ Further records: Bucureşti (Montandon, 1900); Păd. Andronache, 28.VIII.1960 (König, 1975).

Margaritia sticticalis (Linnaeus, 1758): Bucureşti (Montandon, 1900); Pasărea, 21.VII.-15.IX.1997-2009, common.

Ecpyrrhorrhoe rubiginalis (Hübner, 1796): Pasărea, 21.VII.-1.VIII.1997-2008, rare. Further records: Păd. Andronache, 27.VII.1956 (König, 1975).

Pyrausta sanguinalis (Linnaeus, 1761)*: Pasărea, 7.VIII.1997, 1 đ̃.

Pyrausta purpuralis (Linnaeus, 1758): Pasărea, 26.VII.1953 (König, 1975).

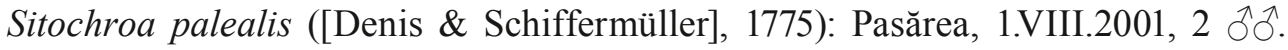
Further records: Comana (Popescu-Gorj, 1964); Păd. Andronache (König, 1975).

Phlyctaenia coronata (Hufnagel, 1767): Pasărea, Putnicu, 21-31.V.1997-2009, common. Further records: Bucureşti (Popescu-Gorj, 1964; König, 1975).

Matuuraia terrealis (Treitschke, 1829): Pasărea, 25.VII.-9.VIII.1997-99, rare. Further records: Bucureşti (Popescu-Gorj, 1964).

Sclerocoma acutella (Eversmann, 1842): Pasărea, 25.VII.-1.VIII.1997-99, rare. Further records: Bucureşti, Snagov (Popescu-Gorj, 1960).

Anania verbascalis ([Denis \& Schiffermüller], 1775)*: Pasărea, 30.V.-9.VIII.19972001, rare.

Ostrinia nubilalis (Hübner, 1796): Pasărea, 1-8.VIII.1996-2010, rare. Further records: Bucureşti (Montandon, 1900; Popescu-Gorj, 1964); Pasărea, 13.VIII.1961 (König, 1975).

Pleuroptya ruralis (Scopoli, 1763): Brăneşti, Pasărea, 21.VII.-9.VIII.1996-2009, common. Further records: Bucureşti (Popescu-Gorj, 1964).

Agrotera nemoralis (Scopoli, 1763): Pasărea, 21.VII.-9.VIII.1997-2008, common. Further records: Bucureşti (Popescu-Gorj, 1964); Băneasa, 10.V.1937 (König, 1975). Nomophila noctuella ([Denis \& Schiffermüller], 1775): Pasărea, Pustnicu, 30.V.2.X.1996-2009, common. Further records: Bucureşti, Comana (Montandon, 1900; Popescu-Gorj, 1964; König, 1975). 


\section{DISCUSSIONS}

Even if tentative, the results are not encouraging, especially if we consider that from the high biodiversity (over 600 species) recorded 100 years ago only a bit more than half were found again in recent times. This does not mean that half of the Lepidoptera fauna of Bucharest has disappeared because the recent research work includes over 180 species that were not reported in the past. We refer first of all to species characteristic to sub-mountainous areas. Such species, even if they existed once, today they cannot find their favorable habitats in Bucharest and its surroundings, such as Lycaena helle ([Denis \& Schiffermüller], 1775), Apatura iris (Linnaeus, 1758), Limenitis populi (Linnaeus, 1758), Erebia ligea (Linnaeus, 1758), Euthrix potatoria (Linnaeus, 1758). Similarly, the species characteristic to sub-mountainous areas reported from the forests of northern Dobrogea around the year 1865 (Mann, 1866) have become extinct in that region. The disappearance of these species is most likely due to climate changes (climate aridisation, which led to the disappearance of such species). On the other hand, these alterations were determined directly by man's intervention through the massive forest clear cuts both in Dobrogea and in Wallachia.

Nowadays, it is relevant the immense poverty of the region in butterflies if compared to the data found in literature. Many old records can be considered as doubtful ones nowadays, e.g.: Lycaena hippothoe (Linnaeus, 1758), Polygonia egea (Cramer, 1775), Melitaea arduinna (Esper, 1784), from the butterflies or Hemaris croatica (Esper, 1779), Menophra abruptaria (Thunberg, 1792) or Staurophora celsia (Linnaeus, 1758) from the nocturnal. Species considered probably extinct, but still to be recorded could be many more, e.g.: Zerynthia polyxena ([Denis \& Schiffermüller], 1775), Kirinia roxelana (Cramer, 1777) or Perisomena caecigena (Kupido, 1825). Thus, a lot of species which were considered "probably extinct" in those areas were recorded again in the period 1996-2010, such as: Catocala dilecta (Hübner, 1808), Plusidia cheiranthi (Tauscher, 1809) or Dolbina elegans Bang-Haas, 1912.

As for the general features of the Lepidoptera-fauna of this region we refer to its main present day features which are the following:

- The Lepidoptera-fauna from this area still preserves some sub-mountainous elements, which lack in the sylvosteppe forests of Dobrogea. Therefore, this region still represents a transition point from the sub-mountainous to that of the steppe and sylvosteppe, e.g.: Aglia tau (Linnaeus, 1758), Moma alpium (Osbeck, 1778), Trisateles emortualis ([Denis \& Schiffermüller], 1775), Xestia rhomboidea (Esper, 1790), etc.

- It still comprises many elements of Anatolian-Balkanic origin, as a conclusion these forests had uninterrupted connection to the forests from the southern Balkan, for example Dolbina elegans, Perisomena caecigena and Noctua haywardi. 
However, the Lepidoptera-fauna underwent through numerous alterations and transformations due to human intervention, the most important being the following ones:

The significant share of the adventive species (imported by means of human activity) and of pest presence. They are dominant in the large majority of the research sites from the surroundings of the capital.

The quite significant share of the steppe-species, since the forest has been restraining continuously in this region and many elements specific to steppe areas are adapting more easily to the farm-ecosystems than the species characteristic to forests.

The antrophic pressure upon the fauna of this region determined some particular features compared to other regions of the country, such as:

The small number of the individuals within the populations of many species, even in the case of species with vigorous populations elsewhere in the country. The reasons are similar to the ones presented before. In some other instances the alteration of environmental factors can determine population gradations of some species (species that are under normal conditions very scarce in other regions of the country) as it was observed with: Marumba quercus ([Denis \& Schiffermüller], 1775), Catocala promissa ([Denis \& Schiffermüller], 1775), Catocala nymphagoga (Esper, 1788), Plagodis pulveraria (Linnaeus, 1758) with more than 100 individuals per night. In the case of butterflies phenomena of this kind could be recorded in 1997-1998 for Neozephyrus quercus (Linnaeus, 1758) with more than 50 individuals per day, this butterfly disappearing completely in the years to come. Even with very rare species we sometimes noticed unexpected population effectives: e.g. Dolbina elegans, with more than 25 individuals per evening on 26-27 July 2001.

Even if the last aspects may represent natural phenomena, the ones presented overleaf are an alarm signal. Therefore we may conclude that the Lepidoptera fauna of this region has altered considerably in the last 100 years, the populations of many species diminished, many species disappeared and transformations occurred in the biology of the species. The region comprises an important number of species protected by European and Romanian laws (Tab. 1). Given the negative changes in habitats presented above, urgent measures are to be taken in order to save the flora and fauna of the region, measures that unfortunately do not seem to be implemented!

\section{ACKNOWLEDGEMENTS}

Thanks to all those who helped to collect material and carry out this study: Valerian Pătraş, Nicolae Vulpe, Nicolae Săvulescu, Aurelian Popescu-Gorj, László Rákosy, Vlad Dincă, Iuliu Szabó, Sorin Marius Stanciu, Robert Görbe, Sándor Lehel Csaba, Dorel Ruşti, Mihai Stănescu, Cristian Mihai, Iorgu Ştefan Ionuţ, Cosmin Ovidiu Manci (Romania), Peter Haneschläger (Austria), Stoyan Beshkov (Bulgaria), István Juhász, Tamás Hácz, Csaba Szabóky (Hungary). 
Taxa protected by law at European or national level.

\begin{tabular}{|c|c|c|c|}
\hline Taxon & $\begin{array}{l}\text { Habitats } \\
\text { Directive } \\
\text { 92/43/EEC }\end{array}$ & $\begin{array}{c}\text { Romania } \\
\text { (national level) } \\
(\text { Rákosy, 2003, 2005) }\end{array}$ & Features \\
\hline Parnassius mnemosyne & $\mathrm{x}$ & & local, very common \\
\hline Euphydryas maturna & $\mathrm{x}$ & & local, very common \\
\hline Lycaena dispar rutila & $\mathrm{x}$ & & local, rare \\
\hline Lopinga achine & $\mathrm{x}$ & & local, common \\
\hline Euplagia quadripunctaria & $\mathrm{x}$ & $\mathrm{x}$ & local, rare \\
\hline Cupido alcetas & & $\mathrm{x}$ & very local, rare \\
\hline Neptis sappho & & $\mathrm{x}$ & very local, common \\
\hline Apatura metis & $\mathrm{x}$ & & local, rare \\
\hline Dolbina elegans & & $\mathrm{x}$ & local, common \\
\hline Plusidia cheiranthi & & $\mathrm{x}$ & very local, rare \\
\hline Zerynthia polyxena* & $\mathrm{x}$ & & Extinct \\
\hline Lycaena helle* & $\mathrm{x}$ & & Extinct \\
\hline Maculinea arion* & $\mathrm{x}$ & & Possible extinct \\
\hline Nymphalis vaualbum* & $\mathrm{x}$ & & Extinct \\
\hline Kirinia roxelana* & & $\mathrm{x}$ & Possible extinct \\
\hline Lycaena hippothoe* & & $\mathrm{x}$ & Extinct \\
\hline Schistostege decussata* & & $\mathrm{x}$ & Possible extinct \\
\hline
\end{tabular}

\section{LEPIDOPTERELE DIN BUCUREŞTI ŞI ÎMPREJURIMI (ROMÂNIA)}

\section{REZUMAT}

Lucrarea reprezintă o sinteză a cunoştinţelor actuale privind fauna de lepidoptere din Bucureşti şi zonele limitrofe pe o rază de $50 \mathrm{~km}$ din jurul capitalei României. Sunt prezentate toate datele despre componenţa faunei de lepidoptere publicate începând cu sfărşitul secolului al XIXlea, precum şi rezultatele noi din ultimii 15 ani. Cercetările întreprinse de autor după 1996 au ca rezultat semnalarea pentru prima dată a prezenței în această regiune a 180 de specii. Chiar dacă cadrul natural din această regiune s-a schimbat radical în secolul XX, ea mai conservă o faună destul de bogată și interesantă. Pădurile adăpostesc bogate populaţii ale sfingidului Dolbina elegans A. Bang-Haas, 1912, una dintre cele mai rare specii de sfingid din Europa, precum şi alte specii de mare valoare faunistică şi zoogeografică ca Noctua haywardi (Tams, 1926) (semnalată pentru prima dată în fauna României din această zonă), Catocala dilecta (Hübner, 1808), Tarachidia candefacta (Hübner, [1831]), Chrysodeixis chalcites (Esper, [1789]), Aedia leucomelas (Linnaeus, 1758) și Hecatera cappa (Hübner, [1809]). De asemenea, este prezentată pentru prima dată şi situaţia actuală a speciilor ocrotite de legislaţia europeană şi naţională din zona capitalei României.

\section{LITERATURE CITED}

ABADJIEV, S., 1995 - Butterflies of Bulgaria, Volume 3, Nymphalidae: Apaturinae \& Nymphalinae, Sofia, $159 \mathrm{pp}$.

ABAFI-AIGNER, L., 1901 - Zur Lepidopteren-fauna Rumäniens. Bulletin de la Société des Sciences, Imprimerie de l'etat, Bucureşti, 9 (5): 1-21.

BURESCH, I., K. TULESCHKOV, 1929 - Die horizontale Verbreitung der Schmetterlinge (Lepidoptera) in Bulgarien, I. Izvestija na tsarskite prirodonauchni instituti v Sofia, 2: 145250. (in Bulgarian) 
BURESCH, I., K. TULESCHKOV, 1930 - Die horizontale verbreitung der Schmetterlinge (Lepidoptera) in Bulgarien. II. Izvestija na tsarskite prirodonauchni instituti v Sofia, 3: 145 248. (in Bulgarian)

CARADJA, A., 1896 - Die Grosschmetterlinge des Königreiches Rumänien, Deutsche Entomologische Zeitschrift, Iris, 9: 1-112. Dresden.

CARADJA, A., 1901 - Die Mikrolepidopteren Rumaniens. Bulletin de la Société des Sciences, 10 (1-2): 110-168.

CARADJA, A., 1903 - Ein Beitrag zur Lepidopteren- Fauna Rumäniens. Bulletin de la Société des Sciences, 12 (1-2): 121-132.

CARADJA, A., 1947 - Macroglossa croatica Esp. besucht Rumänien. Academia Română, Buletinul Secţiunii Ştiinţifice, 30 (1): 61.

CĂPUŞE, I., 1968 - Fam. Tineidae. In: Fauna Republicii Socialiste România (Insecta: Lepidoptera). Edit. Academiei R.S.R., Bucureşti, 11 (9): 1-462. (in Romanian)

DINCĂ, V., 2006 - The Macrolepidoptera (Insecta: Lepidoptera) from Istriţa Hill (Buzău County, Romania). Entomologica Romanica, 10: 5-24.

DRĂGHIA, I., 1970 - Nouvelles contributions à la connaissance des insects mineurs de Bucarest et de ses environs. Travaux du Muséum d'Histoire Naturelle „Grigore Antipa”, 10: 235-240.

DRENOWSKI, A. K., 1907 - Nyakolko novi i s malko nahodishta vidove peperudi (Macrolepidopterae) za Balgariya Periodichesko spisanie na Balgarskoto knizhovno druzhestvo v Sofia, 67 (7-8): 570-607. (in Bulgarian)

DRENOWSKI, A. K., 1930 - Neue Lepidopterenarten aus Bulgarien. Izvestija na bulgarskoto entomologichno druzhestvo v Sofia, 5: 175-188.

EFETOV, K. A., I. V. BUDASHKIN, 1990 - Babocky Krima. Tavria, Simferopol. 110 pp.

FIBIGER, M., 1993 - Noctuidae II. In: Noctuidae Europaea. Entomological Press, Soro. 274 pp.

HACKER, H., A. LEGRAIN, M. FIBIGER, 2008 - Revision of the genus Acontia Ochsenheimer, 1816 and the tribus Acontiini Guenee, 1841 (Old World) (Lepidoptera, Noctuidae, Acontiinae). Esperiana, 14: 7-544.

JOANNIS de. J., 1892 - La liste des Lepidopteres captures par A. Montandon a Bucarest et Comana. Bulletin de la Société Entomologique de France.

KARISCH, T., 1999 - Die Sammlungen des Museums für Naturkunde und Vorgeschichte Dessau. VI. Die Cochylini (Lepidoptera, Tortricidae) der Sammlung Dr. W. Thomas. Naturwissenschaftliche Beiträge des Museums Dessau, 11: 214-234, Museum für Naturkunde und Vorgeschichte Dessau.

KARISCH, T., 2003 - Die Sammlungen des Museums für Naturkunde und Vorgeschichte Dessau. VII. Die Cochylini (Lepidoptera, Tortricidae) der Sammlung Dr. W. Thomas (Nachtrag) mit Beschreibung von zwei neuen Arten. Naturwissenschaftliche Beiträge des Museums Dessau, 15: 121-129, Museum für Naturkunde und Vorgeschichte Dessau.

KARISCH, T., 2004 - Die Sammlungen des Museums für Naturkunde und Vorgeschichte Dessau. IX. Die Tortricini (Lepidoptera, Tortricidae) der Sammlung Dr. W. Thomas. Naturwissenschaftliche Beiträge des Museums Dessau, 16: 70-74, Museum für Naturkunde und Vorgeschichte Dessau.

KARISCH, T., 2010 - Die Sammlungen des Museums für Naturkunde und Vorgeschichte Dessau Die Archipini (Lepidoptera, Tortricidae) der Sammlung Dr. W. Thomas (Teil 1). Naturwissenschaftliche Beiträge des Museums Dessau, 22: 123-131, Museum für Naturkunde und Vorgeschichte Dessau.

KARLSHOLT, O., J. RAZOWSKY, 1996 - The Lepidoptera of Europe. A distributional checklist. Apollo Books, Stenstrup., 308 pp.

KOVÁCS, Z., S. KOVÁCS, 1998 - Familia Eriocraniidae (Lepidoptera) în România. Buletin Informativ, Societatea lepidopterologică română, Cluj-Napoca, 9 (1-2): 9-27. (in Romanian)

KOVÁCS, Z., S. KOVÁCS, 2000 - Familia Incurvariidae (Lepidoptera) în România. Buletin Informativ, Societatea lepidopterologică română, Cluj-Napoca, 11 (1-4): 31. (in Romanian) 
KOVÁCS, Z., S. KOVÁCS, 2001 - Tribul Cochylini (Lepidoptera, Tortricidae) în România. I. (Partea generală Pterochroa, Hysterophora). Buletin Informativ, Societatea lepidopterologică română, Cluj-Napoca, 12 (1-4): 5-45. (in Romanian)

KÖNIG, F., 1975 - Catalogul colecţiei de lepidoptere a Muzeului Banatului. Muzeul Banatului, Timişoara. 284 pp. (in Romanian)

MANN, J., 1866 - Aufzählung der im Jahre 1865 in der Dobrudscha gesammelten Schmetterlinge. Verhandlungen der k. k. zoologisch-botanischen Gesellschaft Wien, 16: 1-40.

MARKOWITSCH, A., 1904 - Materiaux de la faune enthomologique dans les environs de Razgrade. Trudove na bulgarskoto prirodoizpitatelno Druzhestvo v Sofia, 2: 220-252. (in Bulgarian)

MARKOWITSCH, A., 1909 - Peperudnata fauna v Razgradskata okolnost. Sbornik za narodni umotvoren nauka i knizhnina, 25: 1-38. (in Bulgarian)

MONTANDON, A. L., 1900 - Contributions a la faune entomologique de la Roumanie. Bulletin de la Société des Sciences. Bucureşti, 9 (3): 382-386.

NEMEŞ, I., M. VOICU, 1973 - Catalogul colecţiei de lepidoptere "Alexei Alexinschi” de la Muzeul Judeţean Suceava, Part. 3, Superfam: Noctuoidea, Bombycoidea şi Sphingoidea. Studii şi Comunicări, Muzeul Județean Suceava, 4: 1-102. (in Romanian)

NICUlESCU, E. V., 1960 - Fam. Papilionidae. In: Fauna Republicii Populare Române (Insecta: lepidoptera). Edit. Academiei R.P.R. Bucureşti, 11 (5): 1-103. (in Romanian)

NICUlESCU, E. V., 1963 - Fam. Pieridae. In: Fauna Republicii Populare Române (Insecta: lepidoptera). Edit. Academiei R.P.R. Bucureşti, 11 (6): 1-202. (in Romanian)

NICULESCU, E. V., 1965 - Fam. Nymphalidae. In: Fauna Republicii Populare Române (Insecta: lepidoptera). Edit. Academiei R.P.R. Bucureşti, 11 (7): 1-358. (in Romanian)

PITTAWAY, A. R., 2010 - Sphingidae of the Western Palaearctic. http://tpittaway.tripod.com/sphinx/ list.htm.

POLTAVSKY, A. N., K. S. ARTOKHIN, 2006 - Tarachidia candefacta (Lepidoptera: Noctuidae) in the south of European Russia. Phegea, 34 (2): 41-44.

POLTAVSKY, A. N., V. I. SCHUROV, K. S. ARTOKHIN, 2008 - The Introduction, establishment, and spread of the Olive-Shaded Bird-Dropping Moth, Tarachidia candefacta (Hübner, 1831) (Lepidoptera, Noctuidae), in Southern Russia and the Ukraine. Entomological News, 119 (5): 531-536.

POPESCU, A. A., 1938 - Noi contribuţii la fauna Lepidopterelor din Bucureşti şi împrejurimi. Academia Română, Memoriile Secţiunii Ştiinţifice, Seria 3-4 (3): 1-24, București. (in Romanian)

POPESCU, A. A., 1939 - Câteva lepidoptere recoltate în luna august în jurul comunei Mihai Bravu. Extr. Buletinul Societăţii Studenţilor Naturalişti, Anii: 8, 9, 10 (1939): 1-6. (in Romanian)

POPESCU-GORJ, A., 1960 - Lépidoptères nouveaux ou rares pour la faune de la République Populaire Roumaine. Travaux du Muséum d'Histoire Naturelle „Grigore Antipa”, 2: 267-278.

POPESCU-GORJ, A., 1964 - Catalogue de la collection de lépidoptères „Prof. A. Ostrogovich” du Muséum d'Histoire Naturelle „Grigore Antipa”, Bucarest. Edit. Muséum „Grigore Antipa”, Bucarest, 320 pp., 16 pls.

POPESCU-GORJ, A., 1971 - Dolbina elegans, 1912 (Lep. Sphingidae) en Roumanie. Travaux du Muséum d'Histoire Naturelle „Grigore Antipa”, 11: 219-225.

POPESCU-GORJ, A., 1974 - Nouvelles données pour la connaissance de la faune de Lépidoptères de Roumanie. Travaux du Muséum d'Histoire Naturelle „Grigore Antipa”, 15: 213-220.

POPESCU-GORJ, A., 1977 - Nouvelles données pour la connaissance des Lépidoptères de la Fam. Noctuidae de Roumanie. Travaux du Muséum d'Histoire Naturelle „Grigore Antipa”, 18: 141-156.

POPESCU-GORJ, A., 1978 - Colias erate erate Esp. en Roumanie (Lepidoptera: Peridae). Linneana Belgica, 7 (4): 101-116.

POPESCU-GORJ, A., 1986 - Espèces nouvelles ou peu connues dans la faune de Microlépidoptères de Roumanie. Travaux du Muséum d'Histoire Naturelle „Grigore Antipa”, 28: 79-83. 
RÁKOSY, L., 1996 - Die Noctuiden Rumäniens. Stapfhia, Linz, 648 pp.

RÁKOSY, L., 2003 - Lista roşie pentru fluturii diurni din România. Buletin Informativ, Societatea lepidopterologică română, 13 (1-4): 9-26. (in Romanian)

RÁKOSY, L., 2005 - U.E. şi legislaţia pentru protecţia lepidopterelor din România. Buletin de informare Entomologică, Cluj-Napoca, 16 (3-4): 89-96. (in Romanian)

RÁKOSY, L., L. MOMEU, 2009 - Neobiota din România. Presa Universitară Clujeană, Cluj-Napoca, 212 pp. (in Romanian)

RÁKOSY, L., H. NEUMANN, 1990 - Cosmia confinis (Herrich-Schäffer, 1849) şi Chrysodeixis chalcites (Esper, 1789) în fauna României (Lepidoptera, Noctuidae). Studii şi cercetări biologice, Seria biologie animală, Bucureşti, 42 (2): 75-78. (in Romanian)

RÁKOSY, L., M. GOIA, Z. KOVÁCS, 2003 - Catalogul Lepidopterelor României / Verzeichnis der Schmetterlinge Rumäniens. Societatea Lepidopterologică Română, Cluj-Napoca, 446 pp.

SALAY, F., 1910 - Katalog der Makrolepidopteren Rumäniens. Bulletin de la Société des Sciences Bucureşti, 19: 76-206; 453-616.

SCHUROV, V. I., 1998 - Acclimatization of American's bird-dropping moth - The protection and quarantine of plants, 12: 31-32. (in Russian)

SZÉKELY, L., 1994 - Acţiunea schimbărilor condiţiilor de mediu asupra lepidopterofaunei din România. Buletin Informativ, Societatea lepidopterologică română, Cluj-Napoca, 6 (1-2): 27-32. (in Romanian)

SZÉKELY, L., S. M. STANCIU, 2002 - Contribuţii la studiul faunei de lepidoptere din Câmpia Munteniei II. Buletin Informativ, Societatea lepidopterologică română, 12 (1-4): 259-269. (in Romanian)

SZÉKELY, L., P. HANESCHLÄGER, R. GÖRBE, 1998 - Contribuţii la studiul faunei de lepidoptere din Câmpia Munteniei. I. Buletin Informativ, Societatea lepidopterologică română, ClujNapoca, 9 (3-4): 177-186. (in Romanian)

SZÉKELY, L., V. DINCĂ, I. JUHÁSZ, 2011 - Macrolepidoptera from the steppes of Dobrogea (southeastern Romania). Phegea, 39 (3): 85-106.

Received: February 7, 2011

Accepted: December 12, 2011
Săcele, Str.Viitorului $n$ r. 31, sc. B, ap. 9 , RO-505600, Jud. Braşov, România

e-mail: levi.szekely@gmail.com 